\title{
Burning of an hadronic star into a quark or a hybrid star
}

\author{
Alessandro Drago ${ }^{1}$, Andrea Lavagno ${ }^{2}$ and Irene Parenti ${ }^{1}$
}

\begin{abstract}
We study the hydrodynamical transition from an hadronic star into a quark or a hybrid star. We discuss the possible mode of burning, using a fully relativistic formalism and realistic Equations of State in which hyperons can be present. We take into account the possibility that quarks form a diquark condensate. We also discuss the formation of a mixed phase of hadrons and quarks, and we indicate which region of the star can rapidly convert in various possible scenarios. An estimate of the final temperature of the system is provided. We find that the conversion process always corresponds to a deflagration and never to a detonation. Hydrodynamical instabilities can develop on the front. We estimate the increase in the conversion's velocity due to the formation of wrinkles and we find that, although the increase is significant, it is not sufficient to transform the deflagration into a detonation in essentially all realistic scenarios. Concerning convection, it does not always develop. In particular the system does not develop convection if hyperons are not present in the initial phase and if the newly formed quark phase is made of ungapped (or weakly gapped) quarks. At the contrary, the process of conversion from ungapped quark matter to gapped quarks always allows the formation of a convective layer. Finally, we discuss possible astrophysical implications of our results.
\end{abstract}

\section{Introduction}

The possible existence of compact stars partially or totally made of quarks has been proposed many years ago (Witten 1984; Bodmer 1971; Itoh 1970). A likely origin of these compact stellar objects is the conversion of a purely nucleonic (or hadronic) star into a star containing deconfined quark matter through a quark deconfinement phase transition. Several works have discussed when, during the life of the compact star, the deconfinement transition

\footnotetext{
${ }^{1}$ Dip. di Fisica, Univ. di Ferrara and INFN Sez. di Ferrara, I-44100 Ferrara, Italy

${ }^{2}$ Dip. di Fisica, Politecnico di Torino and INFN Sez. di Torino, I-10129 Torino, Italy
} 
should likely take place, either soon after the formation of the neutron star in the supernova explosion (Lugones \& Benvenuto 1998; Benvenuto \& Lugones 1999), or during the cooling of the protoneutron star (Pons et al. 2001). The formation of quark matter could also be delayed, if the deconfinement process takes place through a first order transition so that the purely hadronic star can spend some time as a metastable object (Berezhiani et al. 2003; Drago et al. 2004; Bombaci et al. 2004).

A rather controversial question concerns the duration of the deconfinement process itself. In the first paper discussing this problem (Olinto 1987) it was assumed that the conversion proceeds as a slow combustion and it was found that the velocities involved strongly depend on the temperature of the star and they can be small if the star is hot. Soon after the seminal work of Olinto, Horvath \& Benvenuto (1988) studied the stability of the process and they found that in the presence of gravity the combustion front becomes instable. These authors also stressed that since the appearance of instabilities increases the velocity of the combustion, it is possible that the slow combustion becomes a detonation. On the other hand, no estimate of the velocity of the conversion front, taking into account the instability, was presented and the fluidodynamics equations were written in a non-relativistic frame. The first relativistic calculation of the conversion process was done by Cho et al. (1994). To determine which type of conversion takes place, either a detonation or a deflagration, they studied the conservation of the energy-momentum tensor and of the baryonic flux through the conversion front. Using very simple Equations of State (EoSs) for hadronic matter (the Bethe-Johnson and the Fermi-Dirac EoSs) and the MIT-bag model for quark matter (and without considering the possibility of a mixed phase) they found that the conversion is never a detonation and only for very special values of the parameters it is a slow combustion. For nearly all the parameters' values they obtained an unstable front. In the same relativistic scheme Lugones et al. (1994) studied the case in which the combustion front is preceded by a precompression wave. In this scheme they fixed the velocity of combustion and used the conservation law to determine the final temperature of the combusted phase. Also in this case (as for Cho et al. (1994)) the combusted phase was assumed to be pure strange matter in $\beta$-equilibrium, described by the MIT-bag model and it was not considered the possibility of a mixed phase. In an other work Lugones et al. (2002) found that RayleighTaylor instability can significantly increase the velocity of the combustion; moreover, the presence of a magnetic field can generate a strong asymmetry in the propagation flame, with the maximum velocity in the polar direction.

In our investigation of the conversion process we assume that the formation of quark matter (QM) takes place inside a relatively cold and $\beta$-stable compact star. This scenario is compatible both with the works in which quark deconfinement takes place in a protoneutron star immediately after deleptonization (Pons et al. 2001), and also with the possibility of 
a delayed formation of QM, as discussed in Berezhiani et al. (2003); Drago et al. (2004); Bombaci et al. (2004); Vidana et al. (2005). We will study the conversion problem from the fluidodynamical point of view (Landau \& Lifshitz 1987; Cho et al. 1994), so that it will be possible to determine the type of conversion from nucleonic (or hadronic) matter to QM. Following Landau \& Lifshitz (1987) there are four possibilities: detonation, weak detonation, deflagration (or slow combustion) and strong deflagration. The fastest process is detonation, and in that case the front velocity is suprasonic. It is therefore interesting to explore the possibility that, at least in the case of detonation, the conversion process is so rapid that only strong interactions can take place near the front during the conversion, while weak interactions will restore $\beta$-equilibrium only after the front. The idea to distinguish between fast processes (typically mediated by the strong interaction) and slow processes (due to weak interaction) is common to other physical situations, as, e.g. the computation of viscosity (see Lindblom \& Owen (2002); Drago et al. (2005)). In the literature it has been shown that detonation is difficult to achieve when realistic EoSs are used and matter is assumed to reach $\beta$-equilibrium during the conversion process (Cho et al. 1994). Since the EoS of matter is stiffer if $\beta$-processes are forbidden, then, in principle, it could be easier to obtain a detonation assuming that matter immediately after the front is not yet in $\beta$-equilibrium. In this paper we will consider both the possibility that slow weak interactions take place only after the conversion to QM (which is due to rapid and flavor conserving strong interactions) and also the situation in which $\beta$-equilibrium is immediately restored. It is also important to note that neutrino trapping delays $\beta$-stability. Although in this paper we will not discuss neutrino trapping, an estimate of its importance can be obtained comparing the $\beta$-stable with the not $\beta$-stable scenario. Concerning temperature, one also need to check both possibilities, namely that the detonation front is so rapid that matter immediately after the conversion front is still cold, and the possibility that strong interactions have enough time to thermalize the newly formed phase. The situation is rather different when analyzing deflagration, which is a subsonic process. Since for deflagration heat transmission from the burned to the unburned zone can be crucial, one must also consider burned matter at finite temperature.

The main aim of our work is to study the deconfinement process using realistic EoSs, in particular we will take into account the possibility of forming a mixed phase of quarks and hadrons and we will also discuss the effect of the formation of a diquark condensate. Another important open question that we will investigate is the possibility for convection to develop. As we will show, although the conversion front turns out to be always unstable to gravityinduced Rayleigh-Taylor instability, convection can actually develop only for specific choices of the EoSs, for instance the presence of hyperons in the hadronic phase or the formation of a diquark condensate in the quark phase will help the formation of a convective layer.

The outline of our paper is the following: in Sec. 2 we discuss the fluidodynamics of 
the conversion process; in Sec. 3 we present the EoSs used both for hadronic and for QM and the structure of the resulting compact star. In this section we also discuss the thermal formation of mixed-phase; in Sec. 4 we estimate the temperature reached by the quark (or mixed) phase after deconfinement; in Sec. 5 we show the results of our analysis aiming at classifying the type of conversion; in Sec. 6 we discuss hydrodynamical instabilities and their effect on the conversion velocity; in Sec. 7 we discuss the conditions allowing convection to take place and, finally, in Sec. 8 we summarize our findings and discuss the astrophysical implications of our work.

\section{Fluidodynamics equations of the conversion process}

The starting point of our analysis is given by the fluidodynamics equations describing the conservation of the energy-momentum tensor and of the baryon flux density across the conversion front. Following Landau \& Lifshitz (1987), the continuity equations for the energy-momentum tensor, in the frame in which the front is at rest, read:

$$
\begin{aligned}
\left(e_{h}+p_{h}\right) v_{h} \gamma_{h}^{2} & =\left(e_{q}+p_{q}\right) v_{q} \gamma_{q}^{2} \\
\left(e_{h}+p_{h}\right) v_{h}^{2} \gamma_{h}^{2}+p_{h} & =\left(e_{q}+p_{q}\right) v_{q}^{2} \gamma_{q}^{2}+p_{q}
\end{aligned}
$$

while the continuity of the baryon flux density reads:

$$
\rho_{B}^{h} v_{h} \gamma_{h}=\rho_{B}^{q} v_{q} \gamma_{q}
$$

In the previous equations $e$ is the energy density, $p$ is the pressure, $\rho_{B}$ is the baryon density (all in the rest frame); $v$ is the velocity of a given phase (in the front frame) ${ }^{1}$ and finally $\gamma$ is the Lorentz factor. We use the labels $h$ and $q$ to indicate the hadron and the quark phase, respectively. From Eqs. $(1,2)$ the velocities of the two phases can be obtained and they read:

$$
\begin{aligned}
v_{h}^{2} & =\frac{\left(p_{h}-p_{q}\right)\left(e_{q}+p_{h}\right)}{\left(e_{h}-e_{q}\right)\left(e_{h}+p_{q}\right)} \\
v_{q}^{2} & =\frac{\left(p_{q}-p_{h}\right)\left(e_{h}+p_{q}\right)}{\left(e_{q}-e_{h}\right)\left(e_{q}+p_{h}\right)}
\end{aligned}
$$

Obviously, the velocity of the hadronic phase in the front frame $v_{h}$ equals, but for the sign, the velocity of the front in the frame in which the hadronic phase is at rest. It is possible to

\footnotetext{
${ }^{1}$ Here and in the following we assume the velocity of light $c=1$.
} 
classify the various conversion mechanisms by comparing the velocities of the phases (in the front frame) with the corresponding sound velocities $v_{s}$. The conditions are:

$$
\begin{array}{lll}
v_{h}>v_{s h} & v_{q}<v_{s q} & \text { strong detonation } \\
v_{h}>v_{s h} & v_{q}>v_{s q} & \text { weak detonation } \\
v_{h}<v_{s h} & v_{q}<v_{s q} & \text { weak deflagration } \\
v_{h}<v_{s h} & v_{q}>v_{s q} & \text { strong deflagration. }
\end{array}
$$

It is important to remark that the first condition, namely the relation between the velocity of the hadron phase and the sound velocity of the same phase, can be also replaced by one of the following conditions:

$$
v_{h}>v_{s h} \equiv p_{h}<p_{q} \equiv v_{h}>v_{q}
$$

and similarly for the reversed condition. Let us recall that:

- strong detonation is the process in which a shock wave is responsible for the activation of the burning (this corresponds to what is generally called all simply detonation);

- weak detonation is generally considered impossible to realize in a physical system, because it would imply a heat transmission faster than the velocity of sound (Landau \& Lifshitz 1987);

- weak deflagration is a process in which heat transport is responsible for activating the combustion (this is what is generally called combustion);

- strong deflagration is an unstable process in which the conversion front cannot be described as a simple surface, because instabilities easily take place.

In Sec. 5 we will classify the deconfinement process following the above outlined scheme.

It is important to note that in Eqs. $(1,2,3)$ EoSs at finite temperature have to be used. While in these equations the temperature appear as a parameter, it is possible to relate its value to the heat produced by the deconfinement transition, as discussed in Sec. 4.

A remark is in order. Concerning the actual velocity of the conversion front, it can be determined by solving the fluidodynamics equations only if the conversion is a detonation. If it is a deflagration, other physical scales determine the velocity (significantly reducing its value), as e.g. heat diffusion or the production and diffusion of strangeness. Moreover, if the front is unstable the formation of wrinkles on the front surface or the existence of a convective layer can significantly increase the velocity. In the following we will call deflagrative velocity $v_{d f}$ the velocity obtained solving Eqs. $(1,2,3)$. 


\section{Equations of state of hadrons and quarks}

In this Section we introduce the EoSs that we are going to use in our calculations, both for the hadronic and the quark phase. We discuss the structure of the mixed phase, also taking into account the possibility that $\beta$-equilibrium is not immediately reached after deconfinement. Finally, we present the structure of compact stars obtained using the previous EoSs.

In this Section for simplicity we will discuss EoSs at $T=0$. For the hadronic EoS the extension to finite temperature is well known and rather straightforward (see e.g. Glendenning (1997)). For the quark EoS, it is trivial to compute finite temperature effects for non-interacting QM, much less so for matter in which diquark condensate can take place. We will therefore discuss finite temperature effects only in the case of non-interacting quarks. As it will be clear in the next Sections, our results should not be too much affected by this limitation.

\subsection{EoS of hadronic matter}

Concerning the hadronic phase, we use a relativistic self-consistent theory of nuclear matter in which nucleons interact by exchanging virtual isoscalar and isovector mesons $(\sigma, \omega, \rho)$ (Glendenning \& Moszkowski 1991). At $T=0$, in the mean field approximation and for an infinite and homogeneous system, the thermodynamic potential per unit volume $\Omega$ can be written as:

$$
\Omega=-\frac{1}{3 \pi^{2}} \sum_{B} \int_{0}^{k_{F B}} \mathrm{~d} k \frac{k^{4}}{E_{B}^{\star}(k)}+\frac{1}{2} m_{\sigma}^{2} \sigma^{2}+\frac{1}{3} a \sigma^{3}+\frac{1}{4} b \sigma^{4}-\frac{1}{2} m_{\omega}^{2} \omega_{0}^{2}-\frac{1}{2} m_{\rho}^{2} \rho_{03}^{2},
$$

where the sum runs over the eight baryon species, $E_{B}^{\star}(k)=\sqrt{k^{2}+M_{B}^{\star 2}}$ and the baryon effective masses are $M_{B}^{\star}=M_{B}-g_{\sigma} \sigma$. Only the temporal components of the vector fields (and the third isospin component of $\rho, \rho_{03}$ ) appear at mean field level. Since we will be interested in computing $\beta$-stable matter, let us also write the chemical potentials $\mu_{B}$ which are connected to the effective chemical potentials $\mu_{B}^{\star}$ and to the vector meson fields through the relation:

$$
\mu_{B}^{\star}=\mu_{B}-g_{\omega} \omega-t_{3 B} g_{\rho} \rho_{03},
$$

where $t_{3 B}$ is the isospin 3-component for baryon $B$ and the relation to the Fermi momentum

$k_{F B}$ is provided by $\mu_{B}^{\star}=\sqrt{k_{F B}^{2}+M_{B}^{\star 2}}$. The isoscalar and isovector meson fields $(\sigma, \omega$ and $\rho)$ are obtained as a solution of the field equations in mean field approximation and the related 
couplings $\left(g_{\sigma}, g_{\omega}\right.$ and $\left.g_{\rho}\right)$ are the parameters of the model (Glendenning \& Moszkowski 1991; Knorren et al. 1995).

In Fig. 1 we display the relative concentrations of the various particle species $Y_{i}=\rho_{i} / \rho_{B}$ as a function of baryonic density $\rho_{B}$ by imposing charge neutrality and $\beta$-equilibrium for the GM3 parameter set (Glendenning \& Moszkowski 1991). As it can be seen in the Figure, hyperons start being produced at rather low densities. While this is not a problem in a relativistic mean field model, since stars having a large enough mass can be obtained, in a non relativistic approach the softening of the EoS would be too extreme (Baldo et al. 2000). For this reason, we will discuss both EoSs in which hyperons are taken into account and also pure nucleonic ones.

\subsection{EoS of quark matter}

The central density of compact stellar objects may reach values up to ten times nuclear matter saturation density and therefore it is common opinion that a phase transition to deconfined QM may take place at least in the central region. Recent studies on the QCD phase diagram at finite densities and temperatures have revealed the existence of several possible types of quark phases. Many theoretical works have investigated the possible formation of a diquark condensate in the quark phase, at densities reachable in the core of a compact star (Alford et al. 1999a,b, 2005; Ruster et al. 2006) and the formation of this condensate can deeply modify the structure of the star (Alford \& Reddy 2003; Baldo et al. 2003; Blaschke et al. 2003; Lugones \& Horvath 2002, 2003; Drago et al. 2004; Blaschke et al. 2005; Lavagno \& Pagliara 2005). It is widely accepted that the Color-Flavor Locking (CFL) phase is the real ground state of QCD at asymptoticly large densities. On the other hand, since the structure of the QCD phase diagram depends strongly on the value of the strange quark mass $m_{s}$ and on the diquark coupling parameters, at this stage the complex structure of the QCD phase diagram can not be completely determined at finite densities. In particular, in addition to a phase of unpaired Normal Quark matter (NQ) present at low densities, several superconducting phases (such as 2SC or gapless CFL) can occur at the large baryon densities reached in the center of a compact star.

The transition from hadronic to CFL phase could proceed in two steps, first with a transition from hadronic matter to a $2 \mathrm{SC}$ phase (or to $\mathrm{NQ}$, depending on the model parameters) and then from 2SC to CFL. In the scheme proposed in Drago et al. (2006), the first transition

takes place due to the increase of the baryonic density (due to mass accretion), while the second transition is associated with the deleptonization (and the cooling) of the newly formed star containing 2SC phase. These two transitions can both be first order (Ruster et al. 2006) 
and therefore the newly formed hybrid or quark star containing 2SC quark matter can become metastable and then decay into a star containing CFL phase with a characteristic time delay which corresponds to the nucleation time of a drop of CFL phase inside the 2SC phase. It is interesting to remark that this scenario is compatible with an analysis of the time-structure of the light curves of GRBs (Drago \& Pagliara 2005).

In this paper we consider therefore two different structures for the QM phase: a NQ phase, described by the MIT bag model and a CFL phase. Since we are interested in the bulk properties of a compact star, for the CFL phase we adopt the simple scheme proposed by Alford \& Reddy (2003) where the binding energy $\Delta$ of the diquark condensate in the thermodynamic potential is expanded up to order $(\Delta / \mu)^{2}$ and the gap is assumed to be independent on the chemical potential $\mu$. Following this approximation the thermodynamic potential can be written as:

$$
\Omega_{C F L}=\frac{6}{\pi^{2}} \int_{0}^{\nu} k^{2}(k-\mu) \mathrm{d} k+\frac{3}{\pi^{2}} \int_{0}^{\nu} k^{2}\left(\sqrt{k^{2}+m_{s}^{2}}-\mu\right) \mathrm{d} k-\frac{3 \Delta^{2} \mu^{2}}{\pi^{2}},
$$

with $\nu=2 \mu-\sqrt{\mu^{2}+\frac{m_{s}^{2}}{3}}$, and the quark density $\rho$ is calculated numerically by deriving the thermodynamic potential respect to $\mu$. Pressure and energy density read

$$
\begin{aligned}
P & =-\Omega_{C F L}(\mu)-B-\Omega^{e}\left(\mu_{e}\right), \\
E / V & =\Omega_{C F L}(\mu)+\mu \rho+B+\Omega^{e}\left(\mu_{e}\right)+\mu_{e} \rho_{e} .
\end{aligned}
$$

\subsection{Mixed phase of hadrons and quarks}

The transition from nuclear matter to QM can proceed via a mixed phase if the surface tension at the interface separating quarks and hadrons is not too large (Heiselberg et al. 1993; Voskresensky et al. 2003; Bejger et al. 2005). Gibbs conditions have to be satisfied in the presence of two conserved charges, the baryonic (B) and the electric (C) one (Glendenning (1992), see also Drago \& Lavagno (2001)), whose conservation laws read:

$$
\begin{aligned}
\rho_{B} & =(1-\chi) \rho_{B}^{h}+\chi \rho_{B}^{q}, \\
\rho_{C} & =(1-\chi) \rho_{C}^{h}+\chi \rho_{C}^{q}+\rho_{e}=0 .
\end{aligned}
$$

Here $\chi$ is the fraction of matter in the quark phase and the superscripts $h$ and $q$ label the densities in the hadronic and in the quark phase, respectively. The electron charge density $\rho_{e}$ contributes to make the total electric charge equal to zero.

The EoS appropriate to the description of a compact star has also to satisfy $\beta$-stability conditions. The equations of chemical equilibrium under $\beta$-decay and deconfinement reactions 
are the following:

$$
\begin{array}{ccc}
\mu_{n}-\mu_{p}=\mu_{e} \quad & \mu_{n}-\mu_{p}=\mu_{\mu} \\
2 \mu_{d}+\mu_{u}=\mu_{n} \quad, \quad & \mu_{u}-\mu_{d}=\mu_{p}-\mu_{n}, \\
\mu_{s}= & \mu_{d} .
\end{array}
$$

Finally, the usual condition for mechanical equilibrium, i.e. the equality of the pressure in the two phases, has to be imposed and it reads:

$$
P^{h}=P^{q} .
$$

The previous equations have to be solved together with the field's equations for the adopted hadronic and quark models.

As already mentioned in the Introduction and as it will be further clarified in the next Sections, to our purposes it is also important to consider the EoS of a mixed-phase in which $\beta$-equilibrium has not yet been reached. A crucial analysis has been made in a previous paper (Drago et al. 2005) where it has been studied in detail the process of formation of mixed phase during a perturbation (see in particular Sec. IIIB). The most important result that we can borrow from that analysis, is that it is possible to impose Gibbs conditions, only based on rapid processes, and that through those conditions a mixed phase, stable on the dynamical timescale, can be produced. Indeed, if a detonation can take place the conversion process can be so rapid that $\beta$-equilibrium is reached only after a (short) delay. This non $\beta$-stable mixed-phase is defined to have, at any density, the same isospin ratio $Z / A$ of pure hadronic matter.

In Fig. 2 we show an example of mixed phase EoS. Both the results of the Gibbs and of the Maxwell constructions are displayed. The Gibbs construction corresponds to a vanishing surface tension while the Maxwell construction corresponds to the situation in which the surface tension is so large that it is no more convenient to form finite size structures and no mixed phase would exist inside a compact star. If the surface tension is finite but smaller than $\sigma \approx 30 \mathrm{MeV} / \mathrm{fm}^{2}$ (as estimated by Voskresensky et al. (2003)) the mixed phase shrinks respect to the one obtained using the Gibbs construction and complicated structures have to develop in order to minimize the energy of the system. In Fig. 2 we also indicate a density $\rho_{e q}$ such that for $\rho>\rho_{e q}$ it is energetically convenient to transform completely hadrons into quarks, although the energy of the system can be further reduced forming a mixed phase.

In Fig. 3 we display the relative concentrations of the various particle species $Y_{i}=\rho_{B}^{i} / \rho_{B}$ as a function of baryonic density $\rho_{B}$ by imposing charge neutrality and $\beta$-equilibrium for the GM3 parameter set (Glendenning \& Moszkowski 1991) and using $B^{1 / 4}=180 \mathrm{MeV}$. Quarks 
start being produced, in the mixed phase, at rather low densities. Comparing with Fig. 1, we notice that hyperons density is greatly suppressed and the role of hyperons in neutralizing $\beta$-stable matter is now played mainly by quarks.

\subsubsection{Thermal nucleation of the mixed phase}

For finite values of the surface tension complicated structures develop in the mixed phase, traditionally called drops, rods and slabs. In order to understand under which conditions a fluidodynamical description of the formation of mixed phase is realistic, we have to estimate the dynamical time-scale of the formation of these structures. To this purpose we can borrow again from the analysis of Drago et al. (2005) where two quantities are compared, the size of the barrier which the system has to overcome in order to form a structure and the size of the perturbation of the system. In Drago et al. (2005) the perturbation was due to a gravitational instability and it was estimated to be of the order of a few MeV per baryon. Here the perturbation of the system can be estimated from the size of the mechanical shock (if the process is a detonation) or from the temperature (if it is a deflagration). Since we will see that the conversion always proceeds as a deflagration, we can compare the temperature reached by the system immediately after the conversion with the height of the barrier. If the temperature is not much lower than the height of the barrier the structure formation proceeds thermally and it is very rapid (Olesen \& Madsen 1993, 1994; Di Toro et al. 2006). Instead, new structures can form only via quantum nucleation, typically a very slow process.

The thermal nucleation rate of drops can be estimated as (Olesen \& Madsen 1993, 1994):

$$
\mathcal{R}=\mu^{4} \exp \left(-W_{c} / T\right)
$$

where $\mu$ is the baryon chemical potential and $W_{c}=W\left(R_{c}\right)$ represents the work needed to form the smallest bubble capable of growing. $W_{c}$ corresponds to the maximum of the free energy of the bubble of the new phase. The free energy reads:

$$
W(R)=-\frac{4 \pi}{3} R^{3}\left[\left(P_{2}-P_{1}\right)-\rho_{2}\left(\mu_{2}-\mu_{1}\right)\right]+4 \pi \sigma R^{2}
$$

and its maximum as a function of $R$ (which has to be computed at $\mu_{2}=\mu_{1}$ like in Olesen \& Madsen (1994)) is:

$$
W_{c}=\frac{16 \pi}{3} \frac{\sigma^{3}}{\left(P_{2}-P_{1}\right)^{2}}
$$

which is obtained for a radius $R=R_{c}=2 \sigma /\left(P_{2}-P_{1}\right)$. In Eq. (19) the prefactor has been taken as the chemical potential, while, when thermal nucleation is investigated at very large temperatures, as the ones reached in ultrarelativistic heavy ion scattering, the prefactor is 
related to the temperature. The number of bubbles of new phase formed inside the old phase $^{2}$ in a volume $V$ and in a time $t$ is given by:

$$
\mathcal{N}=\mathcal{R} V t
$$

Let us call $\lambda$ the spacing between two drops in the mixed phase. The number of drops in a volume $V$ is given by $V / \lambda^{3}$. A fluidodynamical description of the formation of mixed phase is realistic if the number of bubbles produced while the front moves over a distance $\lambda$ is of the order of the number of bubbles that have to be present in the mixed phase:

$$
\mathcal{R} V t=\mathcal{R} S \lambda\left(\frac{\lambda}{v}\right) \geq \frac{V}{\lambda^{3}}=\frac{S}{\lambda^{2}} .
$$

Here $v$ is the velocity of the front and $S$ is the area of the surface of the front. Therefore the following constraint has to be satisfied:

$$
\frac{W_{c}}{T} \leq \ln \left(\frac{\mu^{4} \lambda^{4}}{v}\right) \equiv\left(\frac{W_{c}}{T}\right)_{\max } .
$$

For instance, in the case of $\beta$-equilibrium mixed phase and $B^{1 / 4}=165 \mathrm{MeV}$ we obtain $\left(W_{c} / T\right)_{\max }=23$. For the same parameter in Fig. 4 we display $W_{c}$ as a function of the baryonic density $\rho_{B}$ (for different values of the surface tension $\sigma$ ). In the same figure we also show the temperature $\mathrm{T}$ (calculated as described in Sec. 4) and the product between $\mathrm{T}$ and $\left(W_{c} / T\right)_{\max }$. From this figure it is possible to understand in which density regions the fluidodynamical description is allowed. In the density regions where $W_{c}<T \cdot\left(W_{c} / T\right)_{\max }$ the mixed phase forms without delay, the fluidodynamical description of the transition is correct and matter goes directly into the mixed phase. Instead, where $W_{c}>T \cdot\left(W_{c} / T\right)_{\max }$ the formation of the mixed phase is delayed. In this latter case matter, immediately after the front, can not be in the mixed phase. As an example, let us discuss the case $\sigma=10$ $\mathrm{MeV} / \mathrm{fm}^{2}$ shown in Fig. 4 . We can identify three regions. In the central region, with $\rho_{B}$ between $0.17 \mathrm{fm}^{-3}$ and $0.628 \mathrm{fm}^{-3}, W_{c}<T \cdot\left(W_{c} / T\right)_{\max }$ and the fluidodynamical picture is always allowed. In the region between $0.628 \mathrm{fm}^{-3}$ and $\rho_{2}^{G}$ it is energetically convenient to transform completely hadrons into quarks (although the energy of the system can be further reduced by forming a mixed phase) and the fluidodynamical picture can still be applied by burning completely hadrons into quark. Finally, in the region between $\rho_{1}^{G}$ and $0.17 \mathrm{fm}^{-3}$ it is neither possible to form mixed phase soon after the front nor it is convenient to form pure

\footnotetext{
${ }^{2}$ In the low density region of the mixed phase there are drops of quarks in a medium of hadrons. In the upper density region the situation is reversed. We can still apply the formalism here introduced if we suppose that first pure quark matter forms (where energetically convenient) and immediately after drops of hadrons are produced in the quark matter phase.
} 
quark phase (because $\rho_{B}$ is less then $\rho_{\text {eq }}$ ). Therefore in that region the process of formation of mixed phase can not be described fluidodynamically and it can be rather slow.

It is important to note that this description of thermal nucleation is related to the formation of drops of new phase. If the surface tension $\sigma$ is too large the region where the formation of mixed phase is allowed becomes the region in which we have to form more complicated structures to minimize the energy, like rods or slabs. In this case our simplified picture is not applicable.

In conclusion, if the surface tension vanishes the fluidodynamical description of the transition is realistic down to $\rho_{1}^{G}$. If the surface tension is finite but small (until few ten $\mathrm{MeV} / \mathrm{fm}^{2}$ ) it is realistic but only down to a density larger then $\rho_{1}^{G}$. Finally, if the surface tension is large the fluidodynamical picture can be applied down to $\rho_{\text {eq }}$, but assuming matter to first transform (during the hydrodynamical timescale) to a pure quark matter phase.

The process of absorption of an hadron into the pure quark matter phase could also be described phenomenologically as the fusion of a small bubble of quarks (the hadron) into a much larger bubble. In the density region in which pure quark matter can form the large energy gain should make negligible the role played by surface tension, but for very large values of the latter.

\subsection{Compact star structure}

The EoSs analyzed in the last subsections can be used to compute the structure of compact stars by solving the Tolman-Oppenheimer-Volkoff system of equations.

In Figs. 5,6,7 we show the pressure as a function of the baryon density for the various EoSs discussed in the previous subsections. For the pure hadronic phase we adopt the GM3 parameters of Glendenning \& Moszkowski (1991), either using only nucleonic degrees of freedom (label H) or including hyperons (label Hy). Concerning the pure quark phase, we consider both NQ in $\beta$-equilibrium (label uds) and a CFL phase (uds- $\Delta$ ) in which a constant value of the gap has been used $(\Delta=100 \mathrm{MeV})$. In Fig. 5 the value of the pressure of the vacuum is $B^{1 / 4}=155 \mathrm{MeV}$. If the system is allowed to reach $\beta$-stability, mixed phases cannot be formed for such a low value of $B$. Moreover hyperons are not present in the $\beta$-stable EoSs because they are completely substituted by quarks. On the other hand, if the system has not yet reached $\beta$-stability, mixed phase can exist and in Fig. 5 we show an example of such a phase (label H-ud) formed from nucleonic matter and unpaired quarks. In such a case, only up and down quarks can be present since strangeness is absent in the hadronic phase and we assume that there is no time for weak interactions to take place. In Fig. 6 we assume 
$B^{1 / 4}=165 \mathrm{MeV}$. Here a mixed phase can be formed at moderate densities if the quarks are not gapped, but again we obtain pure QM if the formation of a strongly gapped diquark condensate is taken into account. Finally, in Fig. 7 we use $B^{1 / 4}=185 \mathrm{MeV}$. At variance with Figs. 5,6 here the hyperons can be present together with quarks, at least if the latter do not form a condensate. We will not discuss even larger values of $B$ since, as it will be clear in the following, $B^{1 / 4}=185 \mathrm{MeV}$ is in our scheme the largest value of the pressure of the vacuum for which a rapid formation of a mixed phase of deconfined quarks can take place, if a small, but non vanishing surface tension at the interface between hadrons and quarks is taken into account.

In Figs. 8,9,10 we display the mass-radius relations for the same EoSs discussed above. In Figs. 8,9 we have indicated with dots stars having a same baryonic mass $M_{B}=1.7 M_{\odot}$. The gravitational mass of a purely nucleonic star described by the GM3 model is then $M_{G}=1.53 M_{\odot}$, and for an hyperonic star $M_{G}=1.52 M_{\odot}$. In Fig. 8, using $B^{1 / 4}=155 \mathrm{MeV}$ we obtain a quark star made of unpaired quarks having $M_{G}=1.43 M_{\odot}$ and a quark star made of CFL quarks whose mass is $M_{G}=1.32 M_{\odot}$. Similarly, in Fig. 9, using $B^{1 / 4}=165 \mathrm{MeV}$ we obtain a hybrid star made of nucleons and $\mathrm{NQ}$ with a mass $M_{G}=1.48 M_{\odot}$ and a quark star made of CFL quarks with mass $M_{G}=1.39 M_{\odot}$. Finally, in Fig. 10, using $B^{1 / 4}=185 \mathrm{MeV}$ we obtain only hybrid stars, whose maximum baryonic mass exceeds only slightly $M_{B}=1.5 M_{\odot}$ and we indicate with crosses the stars having that same mass $M_{B}$. We have not displayed the mass-radius line for a star made of a mixed phase of hyperons and ungapped quarks, since it is essentially indistinguishable from the $\mathrm{H}$-uds line. The reason is that also in this case, although hyperons are present in the EoS their contribution to the pressure is very small. Here, fixing $M_{B}=1.5 M_{\odot}$, we obtain $M_{G}=1.37 M_{\odot}$ for the nucleonic star, $M_{G}=1.36 M_{\odot}$ for the hyperonic star, $M_{G}=1.36 M_{\odot}$ for the hybrid star made of ungapped quarks and, finally, $M_{G}=1.33 M_{\odot}$ for the hybrid star made of CFL quarks.

In Figs. 11,12,13 we show the density profiles, computed using the same EoSs and for the three values of $B$. In Figs. 11,12 the profiles correspond to a star having $M_{B}=1.7 M_{\odot}$, while in Fig. 13 the star has $M_{B}=1.5 M_{\odot}$. In Fig. 12 we have indicated with two full dots the position inside the nucleonic and the hyperonic star for which $\rho_{B}=\rho_{e q}$, as defined in Sec. 3.3. In Fig. 11 no dot appears because only quark stars are formed and therefore at all densities it is energetically convenient to transform entirely hadrons into quarks. In Fig. 13 only one open circle appear, in correspondence with the transition from an hyperonic star to a star containing CFL quarks. In all other cases, $\rho_{e q}$ is larger than the maximum density of the corresponding purely hadronic star, indicating that the fluidodynamical description of the transition cannot be applied if the surface tension is not low enough, as discussed in Sec. 3.3.1. 


\section{Temperature of the quark phase}

While we assume in our analysis that the hadronic phase is at $T_{H}=0$, the quark or mixed phase produced by the combustion process should be computed at finite temperature. The reason is that we are interested in exothermic processes for which the variation of internal energy is mainly transformed into heat, while a relatively small fraction of the internal energy goes into kinetic energy of the newly formed phase. In the case of an infinite and homogeneous system, which is obviously a rather poor representation of a star, and in the absence of dissipative processes, the new phase would continue flowing at a finite velocity. In a real star the new phase cannot continue moving at finite velocity towards the center of the star and some extra heat is produced by dissipative processes while the kinetic energy dissipates. The numerical value of the kinetic energy turns out to be rather small when compared to the heat directly released by the deconfinement transition (a correction of the order of a few percent in the not- $\beta$-stable case) and therefore we will neglect this contribution.

An estimate of the temperature $T$ of the new phase can be obtained from thermodynamics first principle. Since the variation of the energy per particle $\Delta(E / A)$ depends also on the temperature of the new phase, the following self-consistent equation has to be solved:

$$
\Delta\left(\frac{E}{A}\right)\left(T, \rho_{B}^{h}\right) \equiv \frac{e_{h}\left(u_{h}, \rho_{B}^{h}, T_{h}\right)}{\rho_{B}^{h}\left(u_{h}\right)}-\frac{e_{q}\left(u_{q}, \rho_{B}^{q}, T\right)}{\rho_{B}^{q}\left(u_{q}\right)}=c_{V}^{q}\left(T-T_{h}\right) .
$$

Here the energy density $e\left(u, \rho_{B}, T\right)$ and the baryon density $\rho_{B}(u)$ are computed at finite velocity $u$ and they read (Tolman 1934):

$$
\begin{aligned}
& e(u)=\left(e+p u^{2}\right) \gamma(u)^{2} \\
& \rho_{B}(u)=\rho_{B} \gamma(u)^{2}
\end{aligned}
$$

where $e$ and $\rho_{B}$ are the rest frame quantities. An exothermic process corresponds to a positive $\Delta(E / A)$ and therefore implies $T>T_{h}$.

Both sides of Eq. (25) transform as energies and the equation is therefore covariant. It is possible to recast Eq. (25) in a form showing more explicitly its relation with thermodynamics first principle:

$$
\left[\frac{e_{h}\left(u_{h}, \rho_{B}^{h}, T_{h}\right)}{\rho_{B}^{h}\left(u_{h}\right)}-\frac{e_{q}\left(u_{q}=u_{h}, \rho_{B}^{q}, T\right)}{\rho_{B}^{q}\left(u_{q}=u_{h}\right)}\right]-\left[\frac{e_{q}\left(u_{q}, \rho_{B}^{q}, T\right)}{\rho_{B}^{q}\left(u_{q}\right)}-\frac{e_{q}\left(u_{q}=u_{h}, \rho_{B}^{q}, T\right)}{\rho_{B}^{q}\left(u_{q}=u_{h}\right)}\right]=c_{V}^{q}\left(T-T_{h}\right) .
$$

Here the first term in square brackets is the variation of the internal energy of the system while the second term is the work done by the system. It is important to stress that all 
our calculations are done keeping the volume constant. Due to this assumption, the only mechanical work is the one associated with the kinetic energy of the new phase. Moreover, no chemical contribution need to be present, because chemical equilibrium is always assumed, due to the decoupling of the time-scales and therefore the stoichiometric equation can be used.

Consistently with the astrophysical scenario discussed in the Introduction, we will assume that the initial temperature of the hadronic phase $T_{h}$ is negligible respect to the final temperature of the quark (or mixed) phase, i.e. $T_{h} \simeq 0$. It can be useful to solve Eq. (25) in the hadron matter rest frame $\left(u_{h}=0\right)$ where quarks flow at a velocity $u_{q}$ which can be obtained from Eqs. (4,5). The solution of Eqs. (1,2,3,25) can be obtained using an iterative procedure in which the quark phase is initially assumed at zero temperature when solving Eqs. $(1,2,3)$ and the new estimate of $T$ is obtained from Eq. (25). In central panel of Fig. 17 we will show the difference between the first and the second iteration, indicating that convergence can be reached.

Finally, let us remark that to describe the flow of the new phase in a real star it would be necessary to solve the hydrodynamical equations describing the evolution of the system in which a combustion front propagates (Tokareva et al. 2005). These are rather complicated partial differential equations which, moreover, should be studied together with the equations describing the dynamical readjustment of the star. In our paper we only give a rough estimate of the temperature of the new phase. As it will be shown in the next Sections, a finite value of the temperature affects only marginally the mode of combustion.

The numerical value of the temperature was estimated by Lugones \& Benvenuto (1998) in a different scheme, obtaining results similar to ours.

\section{Results: deflagration regime}

In this Section we discuss the results obtained studying the fluidodynamics Eqs. $(1,2,3)$ with the models discussed in Section 3. We are interested in answering two questions, namely if a detonation is possible and, if this is not the case, which type of deflagration is obtained. As already mentioned in the Introduction, when discussing the possibility of a detonation it is interesting to discuss also the possibility that matter after the conversion front is not yet $\beta$-stable, since weak processes are slower than the strong reactions taking place during deconfinement. Moreover, the conditions for detonation, Eq. (6), are in principle more easy to satisfy if matter after the conversion front is not yet $\beta$-stable, since in that case its EoS is stiffer (as it can be seen from Figs. 5,6,7) and therefore a larger pressure for the new phase 
can be obtained.

In Fig. 14 we show the results obtained for nucleonic matter considering a not $\beta$-stable phase after the front, while in Fig. 15 we show analogous results in the case of $\beta$-stable matter. Finally, in Fig. 16 we show the results for hyperonic matter assuming $\beta$-stability immediately after the front. In the lower panels of these figures the pressure difference between the uncombusted and the combusted phase is shown. Let us recall that $p_{h}-p_{q}>0$ corresponds to a subsonic process and therefore to a deflagration (Eq. 10). In the central panels we show the energy difference between the two phases in the hadron phase rest frame. To have an exothermic process this difference has to be positive, as discussed in Sec. 4. It is also important to remark that Eqs. $(1,2,3)$ can admit multiple solutions, but only one corresponds to an exothermic process. Finally, in the upper panels we show the difference between the velocity of the combusted phase $v_{q}$ and the sound velocity $v_{s q}$ in the same phase (all the velocities are in units of the velocity of light $c$ ). As long as $v_{q}>v_{s q}$ a strong deflagration is obtained. As it can be seen, in all cases ( $\beta$-stable and not $\beta$-stable one) the conditions for detonation are not fulfilled ${ }^{3}$. In the upper panel of Figs. 15,16 and for the lowest value of $B$ we indicate with a star the density below which quarks form with a negative pressure, i.e. they are mechanically unstable immediately after the conversion front. From the central panel of the same figures one can notice that the deconfinement transition stops being exothermic at a density slightly larger and therefore quarks produced in an real, exothermic process are indeed mechanically stable. On the other hand, from the central panel of Figs. 15,16 one can notice that for $B^{1 / 4}=155 \mathrm{MeV}$ an exothermic process with a geometrical front is possible only for very large densities, reachable only at the center of very massive hadronic stars. But the result of the conversion of such massive hadronic star is not a compact star but a black hole, as it can be seen from Fig. 8. Moreover massive hadronic stars are presumably generated by mass accretion and therefore the deconfinement process would likely take place when the mass of the star is smaller. Therefore we can conclude that for very small values of $B$ the process of conversion is not associated with the formation of a geometrical front. Other conversion mechanisms can then take place, for instance convection, and we will come back to this problem in Sec. 7.

Up to now we have only considered zero temperature QM. On the other hand, the process of thermalization of the newly formed phase takes place through strong interaction and it is therefore possible that immediately after the front matter has already to be considered as

\footnotetext{
${ }^{3}$ From a quantitative viewpoint the conditions of Eq. (10) are more near to be satisfied if matter after the conversion front is assumed to be not $\beta$-stable. Comparing Figs. 14 and 15 it is possible to notice that in the case of $\beta$-stable matter $p_{h}>p_{q}$ for all densities while in the not $\beta$-stable case there is a window of low densities for which $p_{q}>p_{h}$ but there the process becomes endothermic.
} 
thermalized. The new phase's temperature can be estimated from energy conservation, as discussed in Sec. 4 and its value reaches a maximum of a few ten MeV near the center of the star. In Fig. 17 we show the results for a finite temperature of the new phase. Also in this case, a detonation is never obtained.

We have also considered the possibility that heat transport is more rapid than deconfinement and therefore hadronic matter near the conversion front reaches a temperature similar to the one of newly formed QM. In Fig. 18 we show results for this scenario and we conclude that also in this case the front is unstable ${ }^{4}$.

As discussed in Sec. 3.2, it is possible that the formation of diquark condensate takes place not immediately after the deconfinement transition but it is delayed. It is therefore interesting to discuss also the transition from NQ to gapped QM, which in our case we assume to be in a CFL phase. In Fig. 19 we show the results for this transition. Also in this case no detonation is obtained and we are always in the regime of strong deflagration. The maximum central density for a stable ungapped quark star is $1.411 \mathrm{fm}^{-3}$ for $B^{1 / 4}=155$ $\mathrm{MeV}$ and $1.619 \mathrm{fm}^{-3}$ for $B^{1 / 4}=165 \mathrm{MeV}$. Therefore, at least for the central region of stars near the maximum mass configuration a geometrical front can indeed form.

Finally, it is important to remark that the velocity of sound in the center of a compact star is typically of the order of $(0.5 \div 0.8) c$ and the velocity of the deflagrative front $v_{d f}$ (defined in Sec. 2) is marginally lower, $(0.4 \div 0.7) c$.

The main result obtained in this Section is that a detonative regime is never directly reached after imposing the continuity conditions on the front. On the other hand two problems still need to be discussed: the estimate of the actual velocity of the deflagrative front taking into account heat and strangeness diffusion and the effect of hydrodynamical instabilities which in principle can increase the conversion velocity transforming a deflagration into a detonation. These two points are discussed in the next Section.

\section{Hydrodynamic instabilities and effective velocity of the front}

In the previous section we have shown that the conversion process always takes place as a deflagration. In this case it is extremely difficult to estimate the velocity of the conversion front. The velocity is governed by the slowest among the processes which need to take place for the combustion to continue. In the seminal work of Olinto (1987) it was shown that, in

\footnotetext{
${ }^{4}$ Cho and $\mathrm{Ng}$ (Cho et al. 1994) found a little window of densities in which detonation is possible but for rather unrealistic high temperature.
} 
the absence of hyperons, the conversion velocity crucially depends on the rapidity by which strangeness is produced in the quark sector and diffuses into the hadronic sector. The final expression for the velocity, assuming a stable laminar front (as if it was a slow combustion), depends on two quantities:

- the temperature of the system;

- the down-strange asymmetry parameter $a_{0}=\left(\bar{\rho}_{d}-\bar{\rho}_{s}\right) / 2 \rho_{B}$, which is related to the minimum number density of strange quarks $\bar{\rho}_{s}$ for which strange quark matter is absolutely stable. Here $\bar{\rho}_{d}$ and $\bar{\rho}_{s}$ are the number densities of down and strange quarks, respectively.

Olinto only discussed the formation of a pure phase of quark matter. Instead, in our paper we have also considered the possibility of producing a mixed phase of hadrons and quarks. It is actually possible to use the formalism of Olinto by re-interpreting the meaning of $a_{0}$ as the minimum strangeness content for which the conversion process is exothermic. The velocity reads:

$$
v_{\mathrm{sc}}=\frac{2}{\sqrt{g}}\left(\frac{\mu}{T}\right) \mathrm{ms}^{-1}
$$

where $g \simeq 2\left(1-a_{0}\right) / a_{0}^{4}$. If strangeness indeed needs to be produced and diffused (i.e. $a_{0} \neq 1$ ) the typical velocities estimated using Eq. (29) are rather low, of the order of a few $\mathrm{km} / \mathrm{s}$ for $T \sim 0.1 \mathrm{MeV}$. The extreme case $a_{0}=1$ corresponds to the possibility of having an exothermic process in the absence of weak interactions. In that situation the conversion is not delayed by strangeness diffusion and the actual velocity is limited by heat conduction, which was assumed as instantaneous in Olinto's analysis. The characteristic timescale of heat diffusion (assuming a laminar front) reads:

$$
t_{q} \sim c_{q} l^{2} / k_{q}
$$

where $c_{q}$ and $k_{q}$ are the specific heat and the heat conductivity of the quark phase and $l$ is the distance over which heat diffuses. The minimal distance for which Eq. (30) can be applied is of the order of the mean free path of the quarks $\lambda_{q}{ }^{5}$. It is therefore possible to roughly estimate the thermalization timescale as $\tau=t_{q}\left(l=\lambda_{q}\right)$ and, correspondingly, to define a heat diffusion velocity $v_{t h}=\lambda_{q} / \tau$. This estimate is similar to the one done by Olinto concerning the production of strangeness in the quark phase and its diffusion up to the hadronic phase. Here heat is again produced in the quark phase and it has to propagate

\footnotetext{
${ }^{5}$ The quark mean free path is very large, up to $10^{3} \mathrm{fm}$, due to Pauli principle, see e.g. Olinto (1987).
} 
over a distance of the order of $\lambda$ to reach the hadronic phase. Estimates of $c_{q}$ and $k_{q}$ can be found in the literature. In particular, for quarks exchanging perturbative gluons $c_{q}$ has been computed by Iwamoto (1982) and $k_{q}$ by Haensel \& Jerzak (1989) and Baiko \& Haensel (1999). The heat diffusion velocity turns out to be of the order of a few percent of the velocity of light. We are not computing the heat diffusion velocity in the case of CFL quark matter because there a laminar front cannot exist.

As already remark by Horvath \& Benvenuto (1988) the conversion velocity can be significantly increased taking into account hydrodynamical instabilities. Indeed in the previous section we have shown that the conversion is always a strong deflagration and not a slow combustion. Therefore the conversion front is unstable and wrinkles can form.

There are at least two types of hydrodynamical instabilities discussed in literature, the Landau-Darrieus (LD) and the Rayleigh-Taylor (RT) (for an introduction to these problems see Zeldovich et al. (1985)). Both these instabilities can develop when $\Delta e \equiv e_{1}-e_{2}>0$. The LD instability is the one which characterizes the strong deflagration regime $\left(v_{q}>v_{s q}\right.$, see Eq. (9)) and the amplification of the wrinkles on the conversion front is directly due to the conservation of the energy-momentum flux, as imposed by Eqs. $(1,2,3)$. That instability can develop independently on the presence of gravity. At the contrary, the RT instability develops if a gravitational field is present and if the direction of the density gradient is opposite to the direction of the gravitational force.

Due to RT and LD instabilities the area of the conversion front increases. The conversion velocity also increases since all exchanges between the burned and the unburned zone are now more efficient. A way of estimating the effective velocity $v_{\text {eff }}$ is through the introduction of the fractal dimension of the surface (Woosley 1990; Blinnikov et al. 2005; Blinnikov \& Sasorov 2005). The larger is the excess of the fractal dimension respect to the dimension of a spherical front, the huger is the increase of the front velocity respect to the laminar case. In the absence of new dimensional scales between the minimal dimension $l_{\min }$ and the maximal dimension $l_{\max }$ of the wrinkle, $v_{\text {eff }}$ is given by:

$$
v_{\mathrm{eff}}=v_{\mathrm{sc}}\left(\frac{l_{\mathrm{max}}}{l_{\min }}\right)^{D-2} .
$$

Here $D$ is the fractal dimension of the surface of the front and it can be estimated as (Blinnikov et al. 2005; Blinnikov \& Sasorov 2005):

$$
D=2+D_{0} \gamma^{2},
$$

where $D_{0} \sim 0.6$ and $\gamma=1-e_{2} / e_{1}$. In Lugones et al. (2002) the effect of hydrodynamical instabilities on the conversion velocity has been discussed. Following their approach we start 
by taking into account only the RT instability which are supposed to be the dominant ones. In order to give a quantitative estimate of the velocity's increase, one needs to compute $l_{\max }$, $l_{\min }$ and $\gamma$. Clearly enough $l_{\max }$ is of the order of a few $\mathrm{Km}$. Concerning $l_{\min }$ it can be estimated as the minimal size of the wrinkle for which the velocity of the RT growing modes is larger than $v_{\mathrm{sc}}$ :

$$
l_{\min }=\frac{4 \pi e_{q} v_{\mathrm{sc}}^{2}}{g \Delta e}
$$

where $\mathrm{g}$ is the gravitational field:

$$
g(r) \equiv-\frac{1}{e(r)} \frac{d P}{d r}
$$

It is important to note that since $l_{\min }$ has to be smaller than $l_{\max }$ a maximum value for $v_{\mathrm{sc}}$ can be defined. Laminar velocities exceeding $v_{\mathrm{sc}}^{\max }$ can not be further increased by RT instabilities.

In Fig. 20 we show the values of $\gamma$ as a function of the density for various values of $B$ both in the $\beta$-stable and in the not $\beta$-stable scenario. In the not $\beta$-stable case the maximum value of $D-2$ is $\sim 0.12$ (for $\gamma \sim 0.45$ ). Instead, in the $\beta$-stable case, $D-2$ can be as large as $\sim 0.34$.

As an example, let us consider an hadronic star with $M_{G}=1.4 M_{\odot}$ for which the maximum density is $0.51 \mathrm{fm}^{-3}$. For $B^{1 / 4}=155 \mathrm{MeV}$ and in the not $\beta$-stable case we obtain at the center of the star $v_{\text {eff }}=0.48 v_{\mathrm{sc}}^{0.83}$. In this case $v_{\mathrm{sc}}^{\max }=0.056 \mathrm{c}$. For $B^{1 / 4}=165$ $\mathrm{MeV}$ and in the $\beta$-stable case we obtain at the center of the star $v_{\text {eff }}=0.40 v_{\mathrm{sc}}^{0.64}$. In this case $v_{\mathrm{sc}}^{\max }=0.079 \mathrm{c}$. It is clear that RT instability alone cannot increase the conversion velocity above the velocity of sound and therefore they cannot transform a deflagration in a detonation.

It is well known that LD instabilities significantly increase the velocity of the conversion. To estimate their effect one can again resort to the fractal scheme, by substituting $l_{\text {min }}$ with a new minimal scale $l_{\text {crit }}$ below which LD instabilities are suppressed. For instance, in the case of Supernovae type Ia several studies indicate that $l_{\text {crit }} \sim 100 l_{\text {th }}$, where $l_{\text {th }}$ is the thickness of the flame (see e.g. Niemeyer \& Woosley (1997)). If that scheme can be applied also to quark deconfinement, $l_{\text {crit }} \sim 10^{5} \mathrm{fm}$, since it seems reasonable to assume $l_{\text {th }} \sim \lambda_{q} \sim 10^{3} \mathrm{fm}$. Although these estimates are clearly very uncertain, taking into account the smallness of $\gamma$ in the not $\beta$-stable case, the velocity can increase by less than one order of magnitude. The conversion process should therefore remain subsonic but for very special choices of the equation of state parameters. In the $\beta$-stable case the velocity can increase by maybe two orders of magnitude, since the $\gamma$ is larger. On the other hand in that case $v_{\text {sc }}$ is extremely small, as estimated from Eq. (29). Therefore in the $\beta$-stable case it is extremely unlikely 
that the the hydrodynamical instabilities can transform the deflagration into a detonation. Nevertheless they increase the conversion velocity by several orders of magnitude, what is important in astrophysical applications.

\section{Convection}

\subsection{Conditions for the existence of a convective layer}

In the previous Section we have discussed instabilities which can increase the conversion velocity by forming wrinkles on the front surface. Another way of accelerating the burning process is via the formation of a convective layer above the conversion front. Here we discuss under which conditions convection can develop during the process of quark deconfinement.

First let us recall that the energy density of the newly formed QM just after the conversion front is smaller than the energy density of hadronic matter immediately before the front. Therefore blobs of QM can try to penetrate the unburned hadronic matter and in principle convection could instaure. For convection to actually develop, the previous condition is necessary but not sufficient, because the drop of QM is formed at a pressure which is also smaller than the pressure of hadronic matter (Landau \& Lifshitz 1987; Horvath \& Benvenuto 1988). The condition for convection to develop reads:

$$
e\left(P_{B}, S_{B}, Y_{e}^{B}\right)<e\left(P, S, Y_{e}\right)
$$

where $S$ is the entropy and $Y_{e}$ is electron fraction and the suffix $B$ indicates the same quantities for the blob. It is called mixing length the distance traveled by the blob before being so modified by the surrounding medium that condition (35) is no more satisfied.

There are various types of convection. In order for the so-called "quasi-Ledoux" convection to develop, the inequality of Eq. (35) has to be satisfied with $P_{B}=P$, in every point of the convection layer, whose size is actually defined through Eq. (35) itself (for a recent review of hydrodynamical problems see e.g. Wilson \& Mathews (2003)). More explicitly, as soon as the quarks' drop enters the hadronic phase, the pressure of the blob starts equilibrating with the pressure of the surrounding material. If the inequality of Eq. (35) with $P_{B}=P$ is not satisfied, the quasi-Ledoux convection can not develop.

A slightly more general type of convection is associated with the question: how long can the quarks' drop travel inside hadronic matter before the pressure equilibrates? The answer to this question depends mainly on the size of the drop $R_{B}$ because the number of scatterings, due to strong interaction and needed to equilibrate the pressure is of the order of the baryon number of the drop. Therefore the mixing length is also of the order of $R_{B}$. 
As we will show in the next subsection, using realistic EoSs, the most relevant case in which quasi-Ledoux condition is not satisfied corresponds to the situation in which mixed phase is produced. A natural length scale for $R_{B}$ can then be the size of the structures of the mixed phase, which is typically of the order of a few fermis (Heiselberg et al. 1993) and this is also the distance traveled by the drop before strong interactions push the drop back into the quark phase. In conclusion, the quasi-Ledoux convection is the only one we will discuss in the following, because other possible convection mechanisms are suppressed in the system we are discussing here.

In our calculation hadronic matter is taken to be cold while the newly formed quarks are in principle at a finite temperature, which can be estimated as done in Sec. 4. Anyway, in the case of massless quarks a finite temperature plays no role, because the relation between density of energy and pressure is independent on the temperature in the massless limit. Taking into account the finite value of the strange quark mass, the energy-pressure relation is temperature dependent, but we have checked that the effect of the temperature on convection is totally negligible, as it will be clarified in the next subsection ${ }^{6}$. The system we are discussing is rather different from other system in which convection develops due to the finite temperature of the drop. In our case the system is strongly degenerate and temperature plays only a minor role. Therefore the size of the mixing-length in our case is not determined by the heath dissipation of the drop, but only by the time needed for strong interactions to equilibrate the pressure and energy density, squeezing the drop.

\subsection{Convection for realistic EoSs}

In Figs. 21,22,23 we show the results of the analysis of the quasi-Ledoux convection using the EoSs and the compact star profiles discussed in previous sections. In Fig. 21 (where $B^{1 / 4}=155 \mathrm{MeV}$ ) we also show examples of trajectories in the pressure vs energydensity plane of a drop of quarks after its formation. We have two sets of letters describing the trajectory of the quark drop, namely the one corresponding to ungapped and not $\beta$ stable mixed phase (suffix 0) and letters corresponding to CFL quark matter, with suffix $g$. In principle we should also have letters with suffix $\beta$ corresponding to ungapped $\beta$-stable QM, but as discussed in Sec. 5, in that case it is not possible to form a geometrical conversion front for $B^{1 / 4}=155 \mathrm{MeV}$. The original drop of hadronic matter from which quarks have

\footnotetext{
${ }^{6}$ In principle one has also to use an isoentropic EoS to describe the evolution of the structure of the drop inside the hadronic phase. Also in this case we have checked that the effect of the temperature on the energy density of the drop is totally negligible.
} 
formed is indicated with $\mathrm{H}$. With $\mathrm{B}_{0}$ we indicate the energy and pressure of the quarks immediately after deconfinement. As discussed in Sec. 5 the pressure and energy density of quarks are smaller than those of hadrons, in agreement with the characteristics of an unstable deflagration front. Since the density of the quark drop is smaller than the density of the hadronic medium the drop will start moving in the opposite direction of gravity, but, as soon as the drop enters hadronic matter its pressure has to equilibrate with that of hadrons $\left(\mathrm{C}_{0}\right)$. It is clear that in the case of not $\beta$-stable mixed phase convection cannot develop, because while $e\left(\mathrm{~B}_{0}\right)<e(\mathrm{H})$, after pressure equilibration $e\left(\mathrm{C}_{0}\right)>e(\mathrm{H})$ and therefore the drop, immediately after entering hadronic matter, has an energy density larger than the hadronic energy density in the same position and the drop is forced to sink back into the QM region. As noted above, the temperature of the quark phase plays no role here because the quark line in the energy-pressure plane is almost independent on the temperature.

From Figs. 21,22 we notice that convection can never develop if hyperons are not present, independent on the value of $B$, as long as diquark condensate is not formed. Fig. 23 shows that convection can develop if hyperons are present both for $B^{1 / 4}=155 \mathrm{MeV}$ and $B^{1 / 4}=165$ $\mathrm{MeV}$. Convection can not develop for $B^{1 / 4}=185 \mathrm{MeV}$.

In Fig. 21 we also show the effect of the formation of a diquark condensate. The scheme we have in mind is the following:

- the transition from hadronic matter to ungapped QM takes place as described above. The velocity of the conversion front is not very large because convection cannot develop;

- in a random site inside the already formed NQ quarks start gapping. Notice that the formation of a CFL gapped quark drop is delayed due to the need to reach $\beta$ stability (producing strangeness and equilibrating the up and down quark content), to deleptonize and to allow the cooling of the star. Moreover, the transition from ungapped to CFL quark matter appear to be first order (Ruster et al. 2006) and therefore a finite nucleation time has to be taken into account;

- the conversion from ungapped to CFL quarks proceeds as a deflagration, as results from the analysis presented in Fig. 19, but in this case convection can develop. This is clear if one considers a drop of $\beta$-stable NQ (A) which transforms into a drop of CFL quarks and then equilibrate its pressure reaching the point $\mathrm{C}_{g}^{\prime}$, with $e\left(\mathrm{C}_{g}\right)<e(\mathrm{~A})$;

- due to convection, the conversion front separating NQ from CFL phase moves rapidly outwards;

- if the time delay in formation of the first drop of CFL quarks is not too large, the new conversion front can reach the "slow" front separating hadronic matter from NQ. As it 
is clear from Fig. 21, drops of CFL quarks can penetrate hadronic matter, generating convection even inside hadronic matter (as long as the energy of the blob $e_{b}>e\left(\mathrm{~L}_{\mathrm{gH}}^{155}\right)$ ) and making the full conversion process considerably faster. Here and in the following we indicate with $\mathrm{L}$ the endpoint of the convective layer. The lower indexes indicate the phase of which the drop is made and the phase in which it propagates, while the upper index indicates the value of $B^{1 / 4}$.

While the existence of a convective layer during the conversion of unpaired QM into gapped QM is independent of the model parameters, the possibility that this convective layer extends inside the hadronic matter region strongly depends on the specific value of the parameters.

We discuss now the thickness of the superconducting layer $\lambda_{c}$ and the velocity $v_{c}$ by which convection expands. Concerning the first point, it is clear that in our scheme the convective layer extends from the center of the star down to the layer whose energy density is $e(\mathrm{~L})$. Typically, $\lambda_{c}$ is of order of a few km. In Figs. 11, 12 we show the endpoints of the convective layers ${ }^{7}$. With NQ we indicate the end of the layer where drops of ungapped $\beta$-stable quarks can develop convection. Similarly we indicate with CFL the layer in which gapped quarks can develop convection.

Concerning the velocity $v_{c}$ of expansion of the convective front, it can be estimated from the conservation of total energy as:

$$
\frac{1}{2} e_{b}(\mathrm{~L}) \mathcal{V}_{f} v_{c}^{2}=e_{b}(\mathrm{C}) \mathcal{V}_{i} U[r(\mathrm{C})]-e_{b}(\mathrm{~L}) \mathcal{V}_{f} U[r(\mathrm{~L})]
$$

where $U(r)$ if the gravity potential and $\mathrm{e}_{b}(\mathrm{C})$ is the energy density of the blob when its pressure equals the pressure of the surrounding medium and the blob starts being accelerated by the bouyant forces. The initial and final values of the volume $\mathcal{V}$ of the drop of QM are related by baryon number conservation:

$$
\mathcal{V}_{f} \rho_{B}(\mathrm{~L})=\mathcal{V}_{i} \rho_{B}(\mathrm{C})
$$

A simpler equation for $v_{c}$ can be obtained by expanding Eq. (36) up to first order in the energy density difference $\delta e_{b}$. Following Wilson \& Mathews (2003) the equation for $v_{c}$ can then be written as:

$$
\frac{1}{2} e_{b} v_{c}^{2}=\delta e_{b} g R_{c}
$$

where $R_{c}$ is the distance traveled by the blob, i.e. the distance from where it has been created up to the end of the convective layer. The gravitational field $g$ is defined in Eq. (34).

\footnotetext{
${ }^{7}$ For $B^{1 / 4}=185 \mathrm{MeV}$ convection can not develop, as shown in Fig. 13.
} 
Using Eq. (38) we obtained values of the convective velocity ranging from $\sim 10^{4} \mathrm{~km} / \mathrm{s}$ when the drop is produced at relatively low densities to $\sim 10^{5} \mathrm{~km} / \mathrm{s}$ when it is produced near the center of a massive star. Although the convective velocity is large, it is anyway much lower than the sound velocity.

\section{Conclusions and astrophysical implications}

To clarify the astrophysical implications of our work let us discuss two scenarios for the formation of QM in which our formalism can be applied.

In the first scenario the surface tension at the interface between hadrons and quarks is negligible or vanishes, i.e. the star can not become metastable respect to the formation of a drop of QM ( $\sigma$ smaller then a few $\left.\mathrm{MeV} / \mathrm{fm}^{2}\right)$. In this case the formation of QM takes place soon after the supernova explosion. In agreement with the analysis of Pons et al. (2001), QM starts forming when the proto-neutron star has deleptonized and its temperature drops down to a few $\mathrm{MeV}$. At the center of the proto-neutron star a drop of QM can form and, if $B$ is not too small, a finite strangeness content is needed for the deconfinement process to be exothermic. The strangeness content could be already present in the hadronic star due to the formation of hyperons. When the drop starts expanding, the process of conversion can be extremely fast within the layer in which deconfinement is energetically convenient even in the absence of weak processes. In this case the conversion front moves at the velocity of the deflagrative front $v_{\text {df }}$, which approaches the velocity of sound (see Sec. 5). As shown in Figs. 5, 6, only at very large densities the EoS of not $\beta$-stable matter is composed of pure quarks. As the conversion layer moves outward, the front enters the region of mixed phase where $v_{\text {df }}$ decreases till it vanishes at the low density boundary of the mixed phase. Before reaching that point the conversion process involving weak reactions becomes first competitive and then dominant. The diffusion of strangeness is a relatively slow process whose velocity can nevertheless be significantly increased by hydrodynamical instabilities. In this scenario heat diffusion plays a marginal role.

In the alternative scenario the surface tension is larger and the hadronic star can therefore become metastable. In the region in which pure quark matter can form there is no substantial difference with the previous scenario although heat diffusion can be necessary to have a rapid expansion of the pure QM (see discussion at the end of Sec. 3.3.1). When the region of mixed phase is reached, the only way of rapidly producing this new phase is via thermal nucleation. In this scenario the deflagrative velocity is therefore limited by the heat diffusion velocity if strangeness need not to diffuse. Again strangeness diffusion will be crucial to convert the outer layers of the star. 
If the surface tension exceeds few ten $\mathrm{MeV} / \mathrm{fm}^{2}$ the process of formation of mixed phase is extremely slow and it cannot be described using a fluidodynamical scheme.

The main result of our analysis, based on realistic EoSs, is that the conversion from hadronic matter to QM, or to a mixed phase of hadrons and quarks and also the transition from unpaired QM to gapped QM always takes place as a deflagration. This result does not change if a finite temperature of the system is taken into account. In our analysis we have shown that the maximum temperature obtained is $\sim 50 \mathrm{MeV}$ near the center of a massive star. For such a relatively low temperature the system remains strongly degenerate and thermal effects are small. To estimate the increase of the conversion velocity due to hydrodynamical instabilities we have used a fractal scheme. Although the wrinkles which develop on the front surface can significantly increase the conversion velocity, in most realistic cases the process remains subsonic and the transformation from deflagration to detonation does not take place. Concerning the possibility of developing convection, this is possible if hyperons are present and if $B$ is not too large and the mass of the compact star not too small. Convection can also develop if quarks can form a condensate. In particular, in the conversion from ungapped to gapped QM convection always takes place.

Let us now discuss two astrophysical problems in which the type of conversion, either deflagrative or detonative, and the conversion velocity play a crucial role.

\section{Neutron star velocities}

It has been proposed by Bombaci \& Popov (2004) that the high velocities displayed by some neutron stars can be attributed to an asymmetric neutrino emission associated with the formation of QM inside the hadronic star. The origin of this asymmetry could be related to a process of deconfinement starting off the center of the star.

In our analysis we have shown that if hyperons are present or if a diquark condensate forms then convection can develop. The possibility of rapidly transporting hot material to the surface of the star via the formation of a convective layer can indeed be at the origin of strong asymmetries in the conversion process $^{8}$.

\footnotetext{
${ }^{8}$ The model proposed in Bombaci \& Popov (2004) and here discussed has no connection with models in which the kicks are explained as due to parity violating processes in the presence of a strong magnetic field, a mechanism which is known to provide almost no contribution to the neutron star velocity.
} 


\section{Gamma Ray Bursts}

It has been speculated several times (Cheng \& Dai 1996; Bombaci \& Datta 2000; Wang et al. 2000; Ouyed \& Sannino 2002; Berezhiani et al. 2003; Bombaci et al. 2004; Vidana et al. 2005) that the so-called long GRBs can be originated by the conversion of an hadronic star into a star containing deconfined QM, either as a pure phase or in phase in which

quarks are mixed with hadrons. Moreover, as discussed in Drago et al. (2006) (see Sec. 3.2 and the analysis of the time-structure of the light curves of GRBs presented in Drago \& Pagliara (2005)) the conversion process can take place in two steps, with a first transition from hadrons to ungapped (or 2SC) quarks and a second transition in which a CFL phase is produced. In order to associate an emission peak with each of the two transitions, the conversion process must be rapid enough to deposit in a few seconds (or less) a huge energy inside the star. Neutrinos will then transport the energy to the exterior on a time scale of order $(10 \div 20)$ s. Clearly, the result of our calculation provides these large velocities, since the conversion process occurs on a time scale of $(0.1 \div 1)$ s for the first transition in the case of a laminar front and it is much more rapid if the hydrodynamical instabilities are taken into account. The second transition lasts only some $10^{-3} \mathrm{~s}$ due to the formation of a convective layer. If the two processes takes place one after the other it is even possible that the formation of diquark condensate accelerates the conversion process by developing a convective layer inside the hadronic phase.

It is also important to recall that the way in which the conversion to quark matter takes place, either via a detonation or a deflagration, is crucial. It has been shown that the mechanical wave associated with a detonation would expel a relatively large amount of baryon from the star surface (Fryer \& Woosley 1998). In the case of a detonation the region near the surface of the compact star where the electron-photon plasma forms (via neutrinoantineutrino annihilation) would be contaminated by the baryonic load and it would be impossible to accelerate the plasma up to the enormous Lorentz factors needed to explain the GRBs.

\section{Acknowledgments}

It is a pleasure to thank S. Blinnikov, G. Pagliara and R. Tripiccione for many useful discussions. 


\section{REFERENCES}

Alford, M., Kouvaris, C. \& Rajagopal, K. 2005, Phys. Rev. D, 71, 054009

Alford, M. \& Reddy, S. 2003, Phys. Rev. D, 67, 074024

Alford, M., Berges, J. \& Rajagopal K. 1999a, Nucl. Phys. B, 558, 219

Alford, M., Rajagopal, K. \& Wilczek, F. 1999b, Nucl. Phys. B, 537, 443

Baiko, D. A. \& Haensel, P. 2003, Acta Phys. Polon. B, 30, 1097

Baldo, M., Burgio, G. F. \& Schulze, H. J. 2000, Phys. Rev. C, 61, 055801

Baldo, M. et al. 2003, Phys. Lett. B, 562, 153

Bejger, M., Haensel, P. \& Zdunik, J. L. 2005, Mon. Not. Roy. Astron. Soc., 359, 699

Benvenuto, O. G. \& Lugones, G. 1999, Mon. Not. Roy. Astron. Soc., 304, L25

Berezhiani, Z., Bombaci, I., Drago, A., Frontera, F. \& Lavagno, A. 2003, ApJ, 586, 1250

Blaschke, D., Fredriksson, S., Grigorian, H., Oztas, A. M. \& Sandin, F. 2005, Phys. Rev. D, 72,065020

Blaschke, D., Grigorian, H., Aguilera, D. N., Yasui, S. \& Toki, H. 2003, AIP Conf. Proc., 660, 209

Blinnikov, S. Iv., Sasorov, P. V. \& Woosley, S. E. 1995, Space Science Rev., 74, 299

Blinnikov, S. Iv. \& Sasorov, P. V. 1995, Phys. Rev. E, 53, 4827

Bodmer, A. R. 1971, Phys. Rev. D, 4, 1601

Bombaci, I. \& Popov, S. B. 2004, A\&A, 424, 627

Bombaci, I. \& Datta, B. 2000, ApJ, 530, L69

Bombaci, I., Parenti, I. \& Vidana, I. 2004, ApJ, 614, 314

Cheng, K. S. \& Dai, Z. G. 1996, Phys. Rev. Lett., 77, 1210

Cho, H. T., Ng, K. W. \& Speliotopoulos, A. D. 1994, Phys. Lett. B, 326, 111

Di Toro, M., Drago, A., Gaitanos, T., Greco, V. \& Lavagno, A. 2006, Nucl. Phys. A, 775, 102 
Drago, A. \& Lavagno, A. 2001, Phys. Lett. B, 511, 229

Drago, A., Lavagno, A. \& Pagliara, G. 2004, Phys. Rev. D, 69, 057505

Drago, A., Lavagno, A. \& Pagliara, G. 2005, Phys. Rev. D, 71, 103004

Drago, A., Lavagno, A. \& Pagliara, G. 2006, Nucl. Phys. A, 774, 823

Drago, A. \& Pagliara, G. 2005, preprint (astro-ph/0512602)

Fryer, C. L. \& Woosley, S. E. 1998, ApJ., 501, 780

Glendenning, N. K. \& Moszkowski, S. A. 1991, Phys. Rev. Lett., 67,2414

Glendenning, N. K. 1997, Compact Stars (Springer-Verlag)

Glendenning, N. K. 1992, Phys. Rev. D, 46, 1274

Haensel, P. and Jerzak, A. J. 1989, Acta Phys. Polon. B, 20, 141

Heiselberg, H., Pethick, C. J. \& Staubo, E. F. 1993, Phys. Rev. Lett., 70, 1355

Horvath, J. E. \& Benvenuto, O. G. 1988, Phys. Lett. B, 213, 516

Itoh, N. 1970, Prog. Theor. Phys., 44, 291

Iwamoto, N. 1982, Ann. Phys., 141, 1

Knorren, R., Prakash, M. \& Ellis, P. J. 1995, Phys. Rev. C, 52, 3470

Landau, L. D. \& Lifshitz, E. M. 1987, Fluid Mechanics (Pergamon Press)

Lavagno, A. \& Pagliara, G. 2005, preprint (nucl-th/0504066), in press on Int. J. Mod. Phys. D

Lindblom, L. \& Owen, B. J. 2002, Phys. Rev. D, 65, 063006

Lugones, G. \& Benvenuto, O. G. 1998, Phys. Rev. D, 58, 083001

Lugones, G., Benvenuto, O. G. \& Vucetich, H. 1994, Phys. Rev. D, 50, 6100

Lugones, G., Ghezzi, C. R., de Gouveia Dal Pino, E. M. \& Horvath, J. E. 2002, ApJ, 581, L101

Lugones, G. and Horvath, J. E. 2002, Phys. Rev. D, 66, 074017

Lugones, G. and Horvath, J. E. 2003, A\&A, 403, 173 
Olesen, M. L. \& Madsen, J. 1993, Phys. Rev. D, 47, 2313

Olesen, M. L. \& Madsen, J. 1994, Phys. Rev. D, 49, 2698

Olinto, A. 1987, Phys. Lett. B, 192, 71

Ouyed, R. \& Sannino, F. 2002, A\&A, 387, 725

Pons, J. A., Steiner, A. W., Prakash, M. \& Lattimer, J. M. 2001, Phys. Rev. Lett., 86, 5223

Ruster, S. B., Werth, V., Buballa, M., Shovkovy, I. A. \& Rischke, D. H. 2006, Phys. Rev. D, 73,034025

Tokareva, I., Nusser, A., Gurovich, V. \& Folomeev, V. 2005, Int. J. Mod. Phys. D, 14, 33

Tolman, R. 1934, Relativity Thermodynamics and Cosmology (Oxford University Press)

Vidana, I., Bombaci, I. \& Parenti, I. 2005, J.Phys. G, 31, S1165

Voskresensky, D. N., Yasuhira, M. \& Tatsumi, T. 2003, Nucl. Phys. A, 723, 291

Wang, X. Y., Dai, Z. G., Lu, T., Wei, D. M. \& Huang, Y. F. 2000, A\&A, 357, 543

Wilson, J. R. \& Mathews, G. J. 2003, Relativistic Numerical Hydrodynamics (Cambridge University Press)

Witten, E. 1984, Phys. Rev. D, 30, 272

Woosley, S. E. 1990, in Supernovae, edited by A. G. Petschek (Springer-Verlag, New York, 1990), p.182

Niemeyer, J. C. \& Woosley, S. E. 1997, ApJ, 475, 740

Zeldovich, Ya. B., Barenblatt, G. I., Librovich, V. B. \& Makhviladze, G. M. 1985, The Mathematical Theory of Combustion and Explosions (Plenum, New York, 1985) 


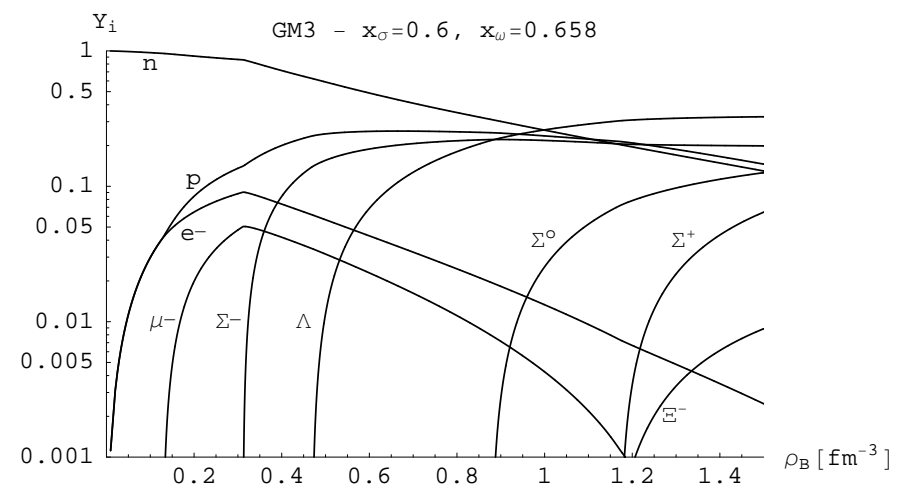

Fig. 1. - Particle fractions $Y_{i}$ of neutral and $\beta$-stable hadronic matter as a function of baryonic density $\rho_{B}$ for the GM3 hadronic equation of state of Glendenning \& Moszkowski (1991).

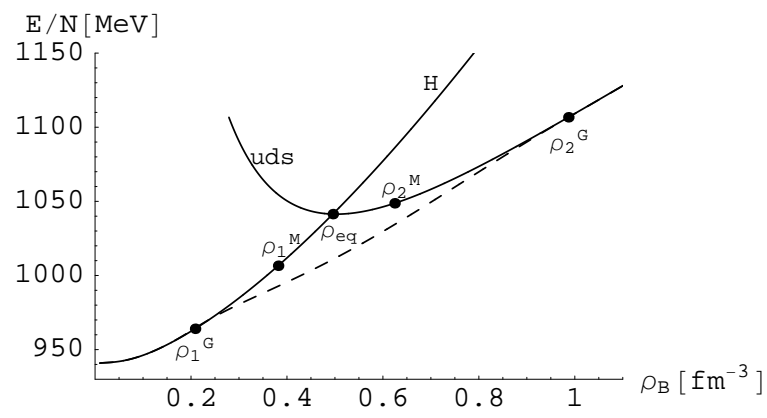

Fig. 2.- Typical scheme of a first order transition at finite density with mixed phase. The solid lines represent the pure hadronic phase (H) and the pure quark phase (uds). The dashed line, starting at $\rho_{1}^{G}$ and ending at $\rho_{2}^{G}$, is the mixed phase obtained imposing Gibbs conditions and for a vanishing surface tension. When the surface tension increases the region of mixed phase shrinks and it reduces to the one obtained using the Maxwell construction which starts at $\rho_{1}^{M}$ and ends at $\rho_{2}^{M} . \rho_{e q}$ is the density at which the energies of the two pure phases are equal. 


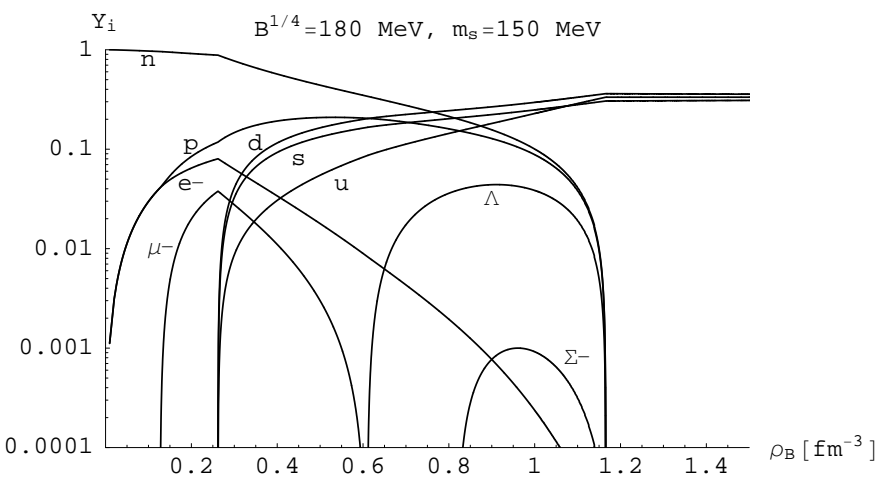

Fig. 3.- Particle fractions $Y_{i}$ of neutral and $\beta$-stable hadronic and quark matter as a function of baryonic density $\rho_{B}$ for the same GM3 hadronic equation of state used in Fig. 1 and using the MIT bag model with $B^{1 / 4}=180 \mathrm{MeV}$ to describe the quark phase.

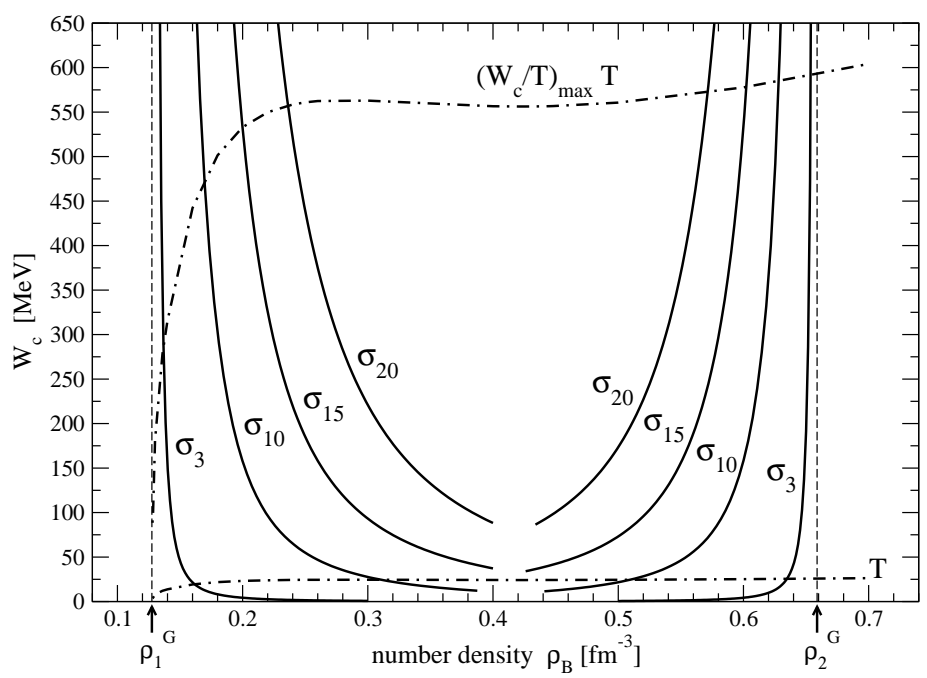

Fig. 4.- Work needed to form a bubble of new phase as a function of baryonic density $\rho_{B}$ (solid lines). The four lines at lower density correspond to the formation of bubbles of quarks in the hadronic medium (for four different values of the surface tension $\sigma$ ). The four lines at higher density correspond to the formation of bubbles of hadrons in a pure quark matter medium. Here we have used the GM3 EoS to describe the hadronic phase and the MIT bag model with $B^{1 / 4}=165 \mathrm{MeV}$ to describe the quark phase. $\beta$-stability has been imposed in both phases. The starting and the ending point of the mixed phase using the Gibbs construction are shown $\left(\rho_{1}^{G}\right.$ and $\left.\rho_{2}^{G}\right)$. We also show the temperature $\mathrm{T}$ reached by the system due to the exothermic deconfinement process and the product between $\mathrm{T}$ and $\left(W_{c} / T\right)_{\max }$, indicating the maximum value of the work for which thermal nucleation can take place and be rapid. 


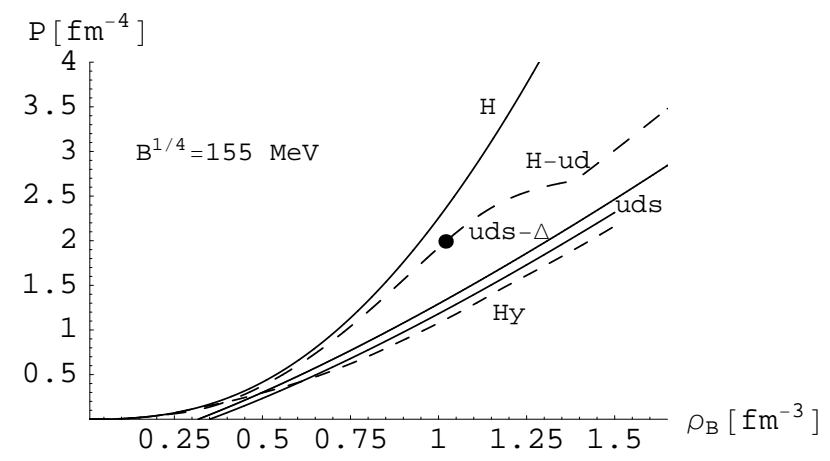

Fig. 5. - Pressure as a function of the baryon density for pure nucleonic phase $(\mathrm{H})$, not $\beta$ stable mixed phase of nucleonic matter and unpaired quark ud matter (H-ud), unpaired uds quark matter (uds), pure CFL phase (uds- $\Delta$ ). The dot on the not $\beta$-stable EoS corresponds to $\rho_{e q}$, defined in Fig. 2. Here $B^{1 / 4}=155 \mathrm{MeV}$.

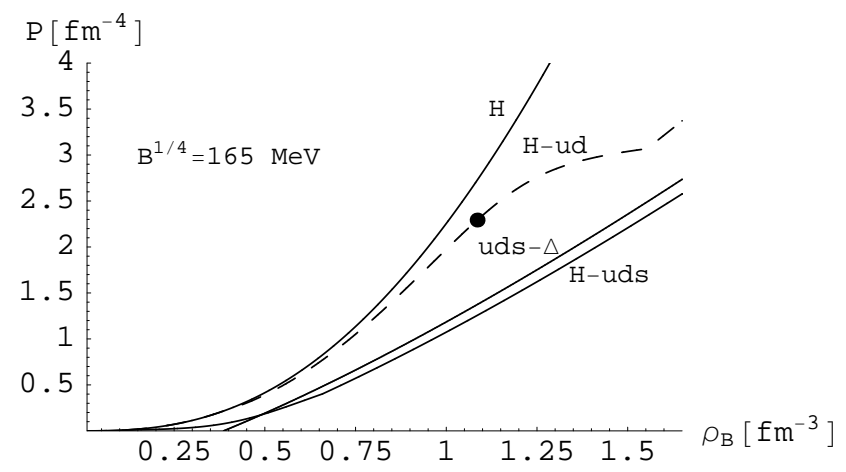

Fig. 6. - Pressure as a function of the baryon density for pure nucleonic phase $(\mathrm{H})$, not $\beta$-stable mixed phase of nucleonic matter and unpaired quark ud matter (H-ud), $\beta$-stable (mixed) phase of hadronic and unpaired uds quark matter (H-uds), pure CFL phase (uds$\Delta)$. The dot on the not $\beta$-stable EoS corresponds to $\rho_{e q}$, defined in Fig. 2. Here $B^{1 / 4}=$ $165 \mathrm{MeV}$. 


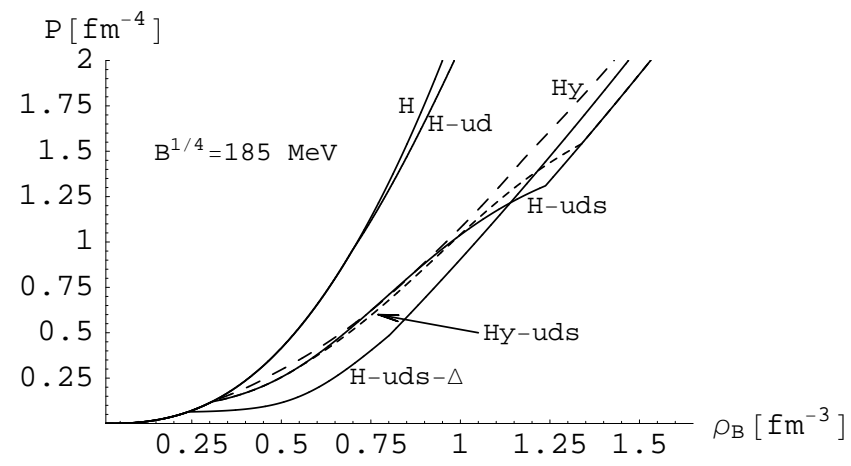

Fig. 7.- Pressure as a function of the baryon density for pure nucleonic phase $(\mathrm{H})$, hyperonic matter (Hy), not $\beta$-stable mixed phase of nucleonic matter and unpaired quark ud matter (H-ud). Also displayed are $\beta$-stable (mixed) phases made of nucleons and unpaired uds quarks (H-uds), of hyperons and unpaired uds quarks (Hy-uds) and, finally, of nucleons and CFL quarks (H-uds- $\Delta$ ). Here $B^{1 / 4}=185 \mathrm{MeV}$.



Fig. 8.- Mass-radius relations of nucleonic stars $(\mathrm{H})$, hyperonic stars (Hy), quark stars made of unpaired quark matter (uds) and of color superconducting CFL phase (uds- $\Delta$ ). The dots indicate stars whose baryonic mass is $M_{B}=1.7 M_{\odot}$. Here $B^{1 / 4}=155 \mathrm{MeV}$. 


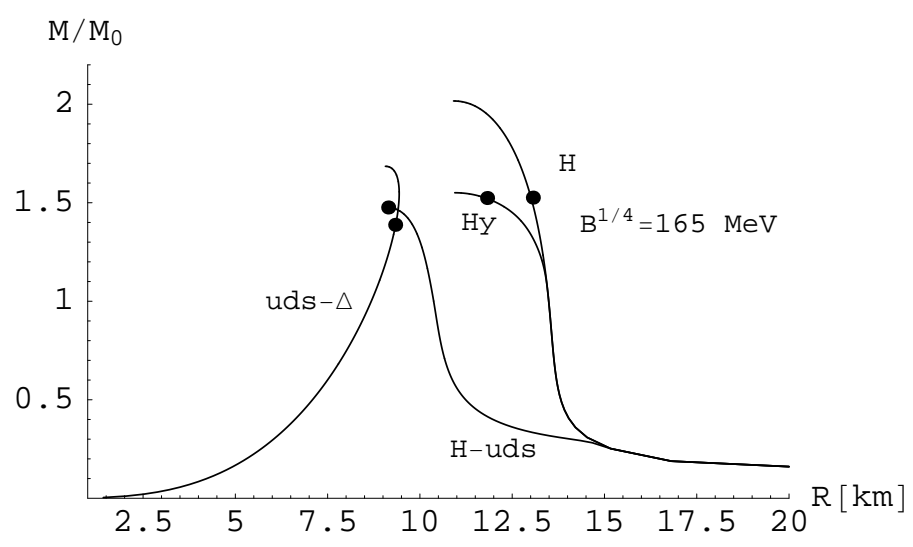

Fig. 9.- Mass-radius relations of nucleonic stars $(\mathrm{H})$, hyperonic stars (Hy), hybrid stars made of hadrons and of unpaired quark matter (H-uds) and quark stars made of color superconducting CFL phase (uds- $\Delta$ ). The dots indicate stars whose baryonic mass is $M_{B}$ $=1.7 M_{\odot}$. Here $B^{1 / 4}=165 \mathrm{MeV}$.



Fig. 10.- Mass-radius relations of nucleonic stars (H), hyperonic stars (Hy), hybrid stars made of hadrons and of unpaired quark matter (H-uds) and of hadrons and CFL quarks $($ H-uds- $\Delta)$. The crosses indicate stars whose baryonic mass is $M_{B}=1.5 M_{\odot}$. Here $B^{1 / 4}=$ $185 \mathrm{MeV}$. 


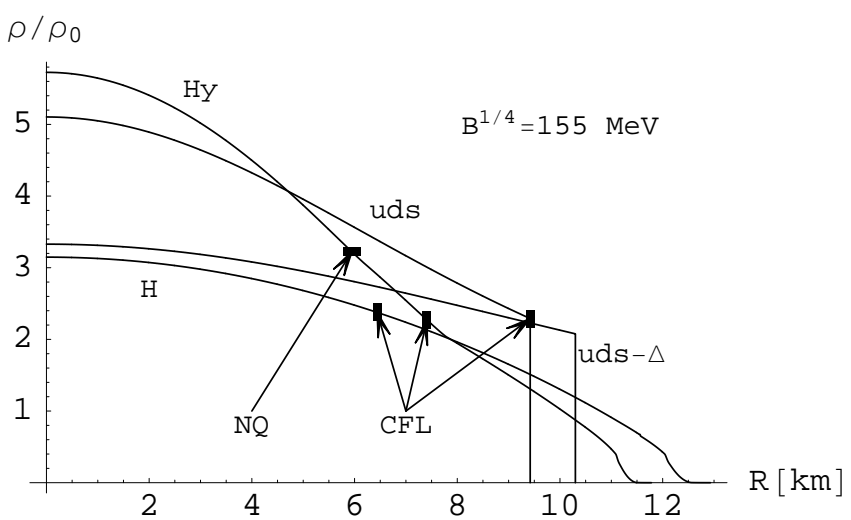

Fig. 11. - Baryon density profiles for different compact stars having the same baryonic mass $M_{B}=1.7 M_{\odot}$. Here $B^{1 / 4}=155 \mathrm{MeV}$. Labels as in previous figures. The arrows indicate the endpoints of the convective layers (see Sec. 7.2).

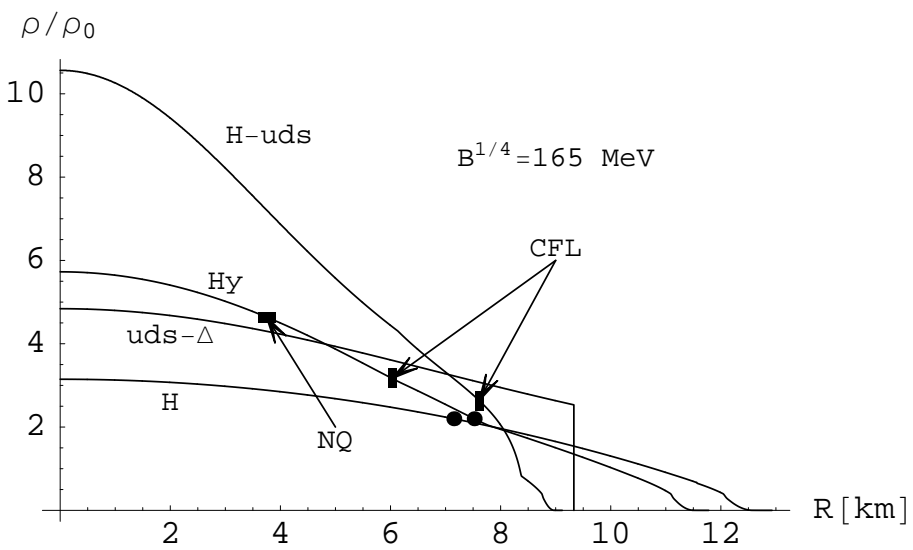

Fig. 12.- Baryon density profiles for different compact stars having the same baryonic mass $M_{B}=1.7 M_{\odot}$. Here $B^{1 / 4}=165 \mathrm{MeV}$. Labels as in previous figures. The dots indicate $\rho_{e q}$ for a transition from nucleonic or hyperonic matter to unpaired quark matter. When the transition is to CFL quark matter, a quark star is obtained and the hydrodynamical argument can be applied to all regions of the star. The arrows indicate the endpoints of the convective layers (see Sec. 7.2). 




Fig. 13. - Baryon density profiles for different compact stars having the same baryonic mass $M_{B}=1.5 M_{\odot}$. Here $B^{1 / 4}=185 \mathrm{MeV}$. Labels as in previous figures. The open circle indicate $\rho_{e q}$ for a transition from hyperonic matter to CFL quark matter. In all other cases the central density of the hadronic star was lower than $\rho_{e q}$. 


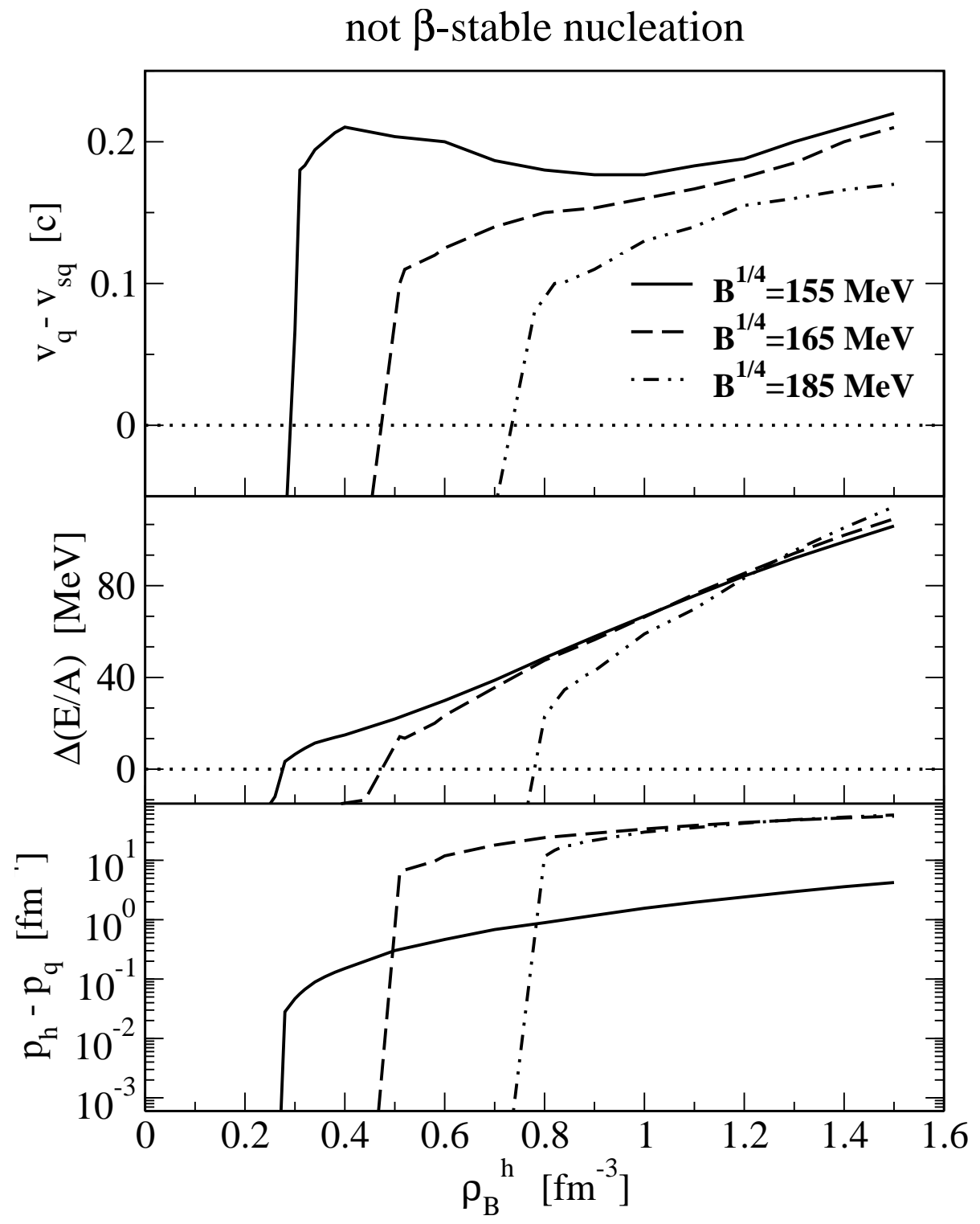

Fig. 14. - Upper panel: difference between the velocity $v_{q}$ of the combusted phase and the sound velocity $v_{s q}$ in the same phase. All the velocities are in units of the velocity of light and in the front frame. The three lines corresponds to different values for the bag constant. Center panel: energy difference between the two phases (in the hadron phase rest frame), as defined in Eq. (25). Lower panel: pressure difference between the uncombusted and the combusted phase. Here the combusted phase is not $\beta$-stable. 


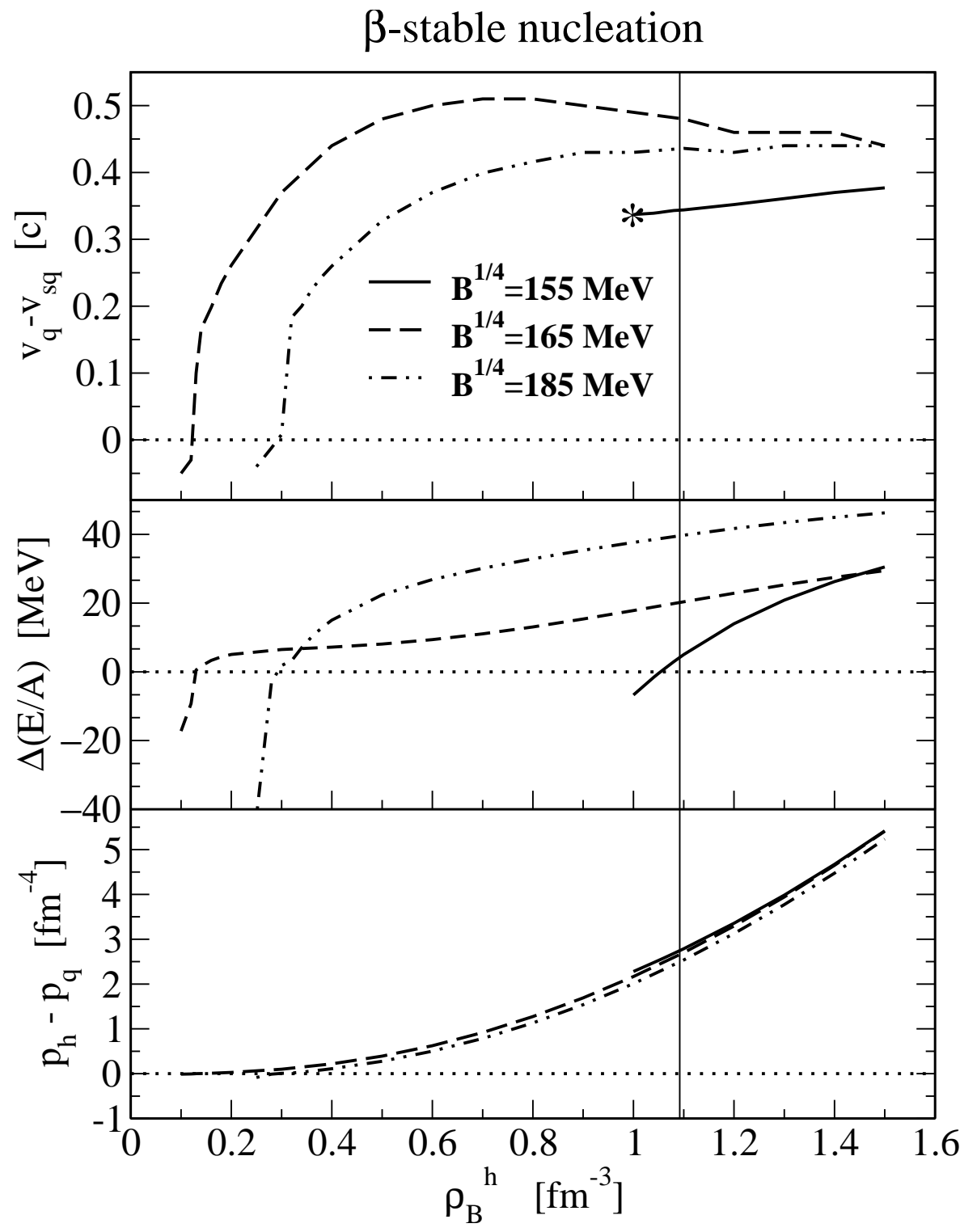

Fig. 15.- Same as in Fig. 14. Here the combusted phase is $\beta$-stable. The star in the upper panel indicates the density below which the pressure of the newly formed quark matter is negative. The vertical line corresponds to the central density of the most massive stable configuration of a nucleonic star obtained using GM3 model $\left(\rho_{h}^{\max }=1.09 \mathrm{fm}^{-3}\right)$. 


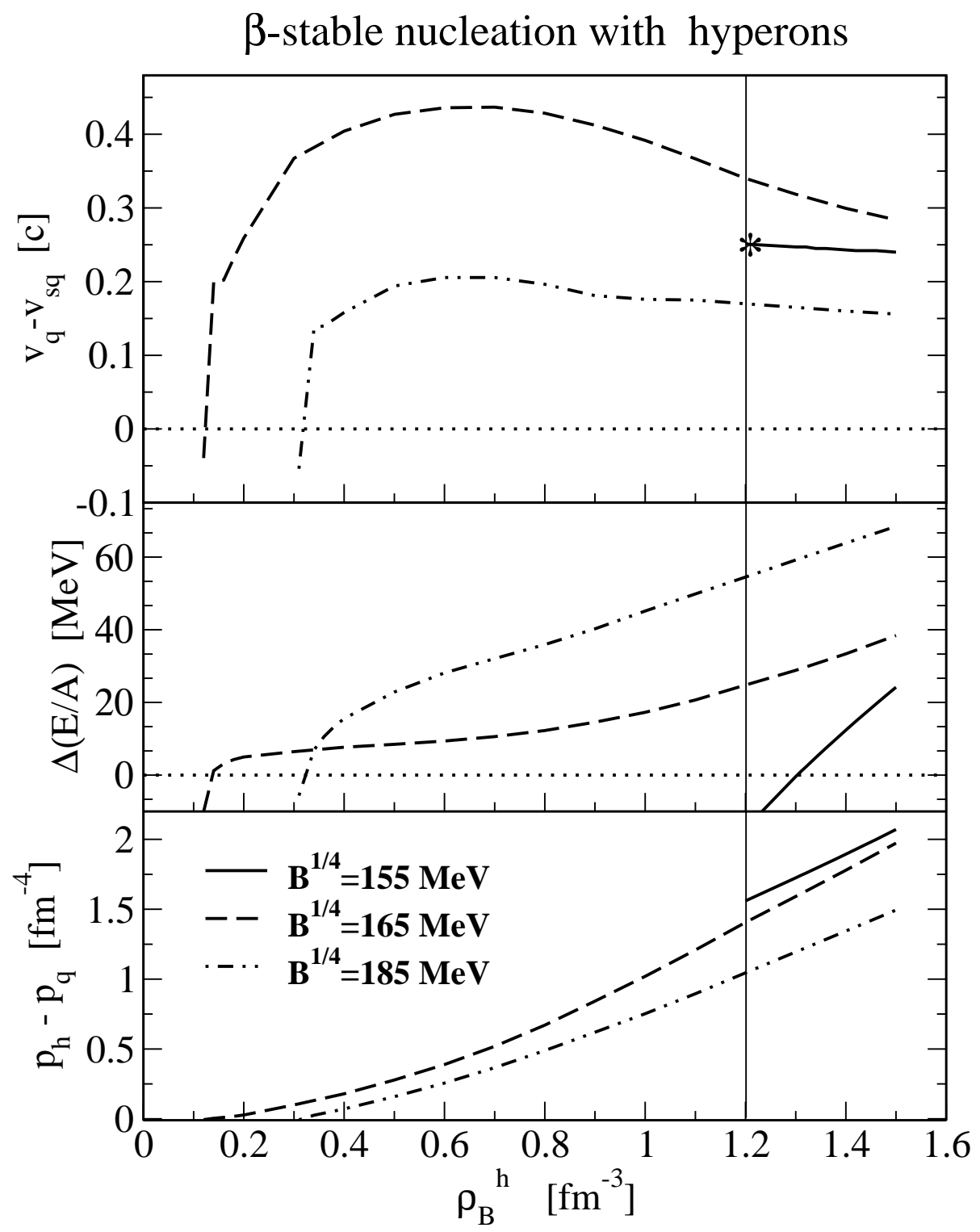

Fig. 16. - Same as in Fig. 15. Here hyperons are included in the hadronic phase. The combusted phase is again $\beta$-stable. The vertical line corresponds to the central density of the most massive stable configuration of a hyperonic star obtained using GM3 model $\left(\rho_{h}^{\max }=1.20 \mathrm{fm}^{-3}\right)$. 


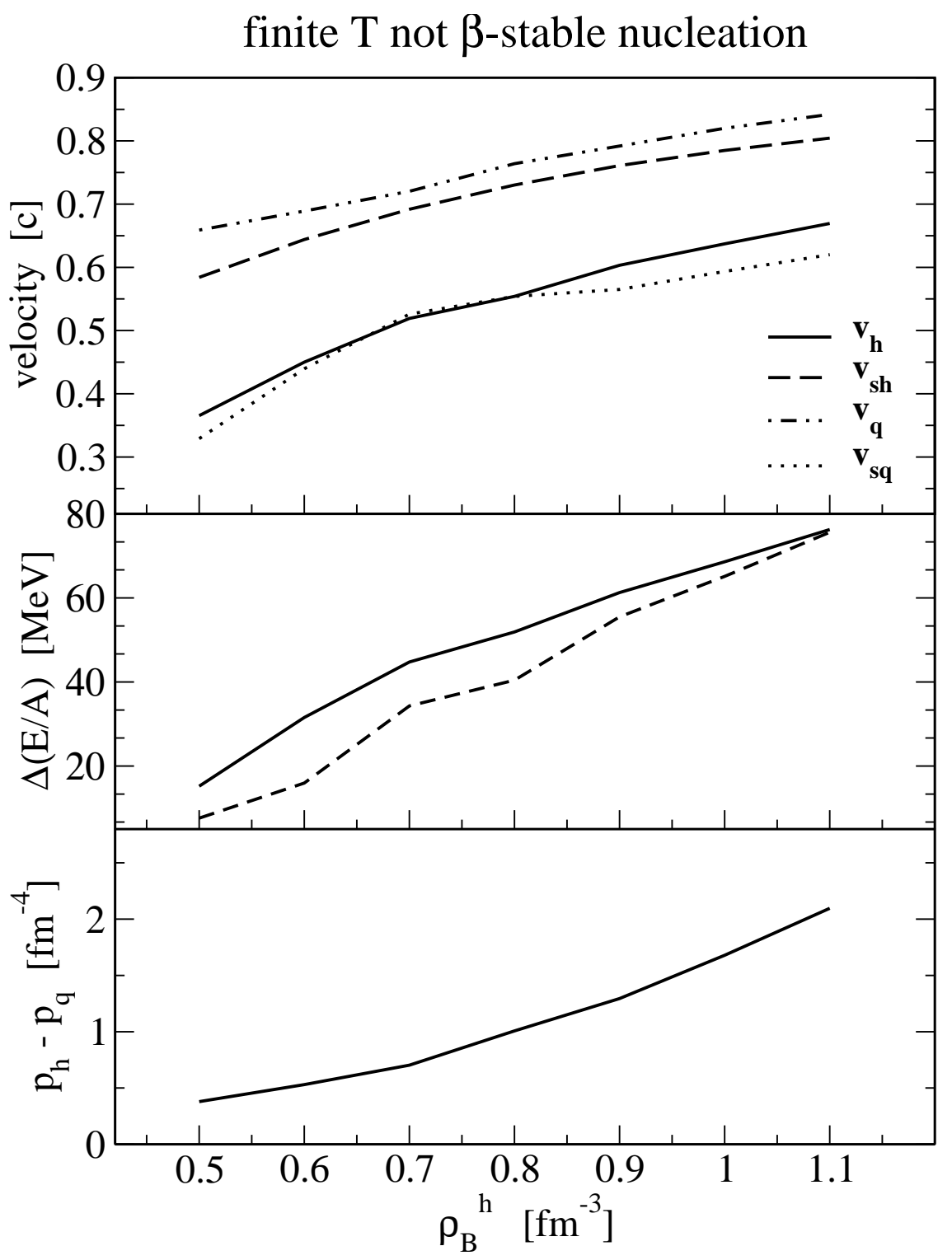

Fig. 17.- Upper panel: velocity of hadronic phase $v_{h}$, of the burned phase $v_{q}$ and corresponding sound velocities $v_{s h}$ and $v_{s q}$, all in units of the velocity of light and in the front frame. Center panel: energy difference between the two phases (in the hadron phase rest frame). The dashed and the solid lines correspond to the first and to the second iteration in the solution of Eqs. $(1,2,3,25)$. Lower panel: pressure difference between the uncombusted and the combusted phase. Here the combusted phase is obtained using $B^{1 / 4}=170 \mathrm{MeV}$, temperatures from 5 to $40 \mathrm{MeV}$ (as estimated from the solid line in the central panel) and it is not $\beta$-stable. 


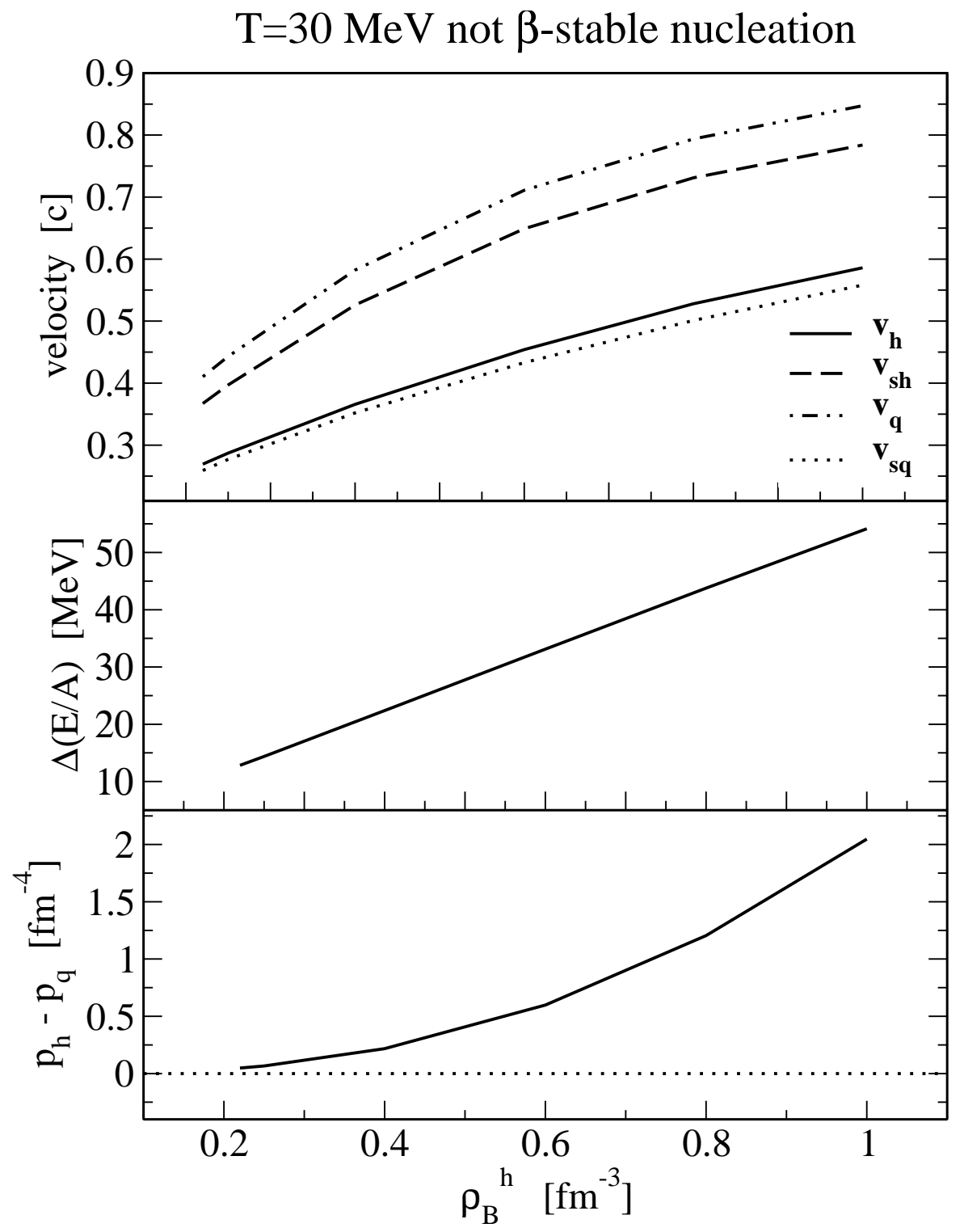

Fig. 18. - Notations as in Fig. 17. The combusted phase is obtained using $B^{1 / 4}=170$ $\mathrm{MeV}$. Here both the quark and the hadronic phase are at $\mathrm{T}=30 \mathrm{MeV}$. 


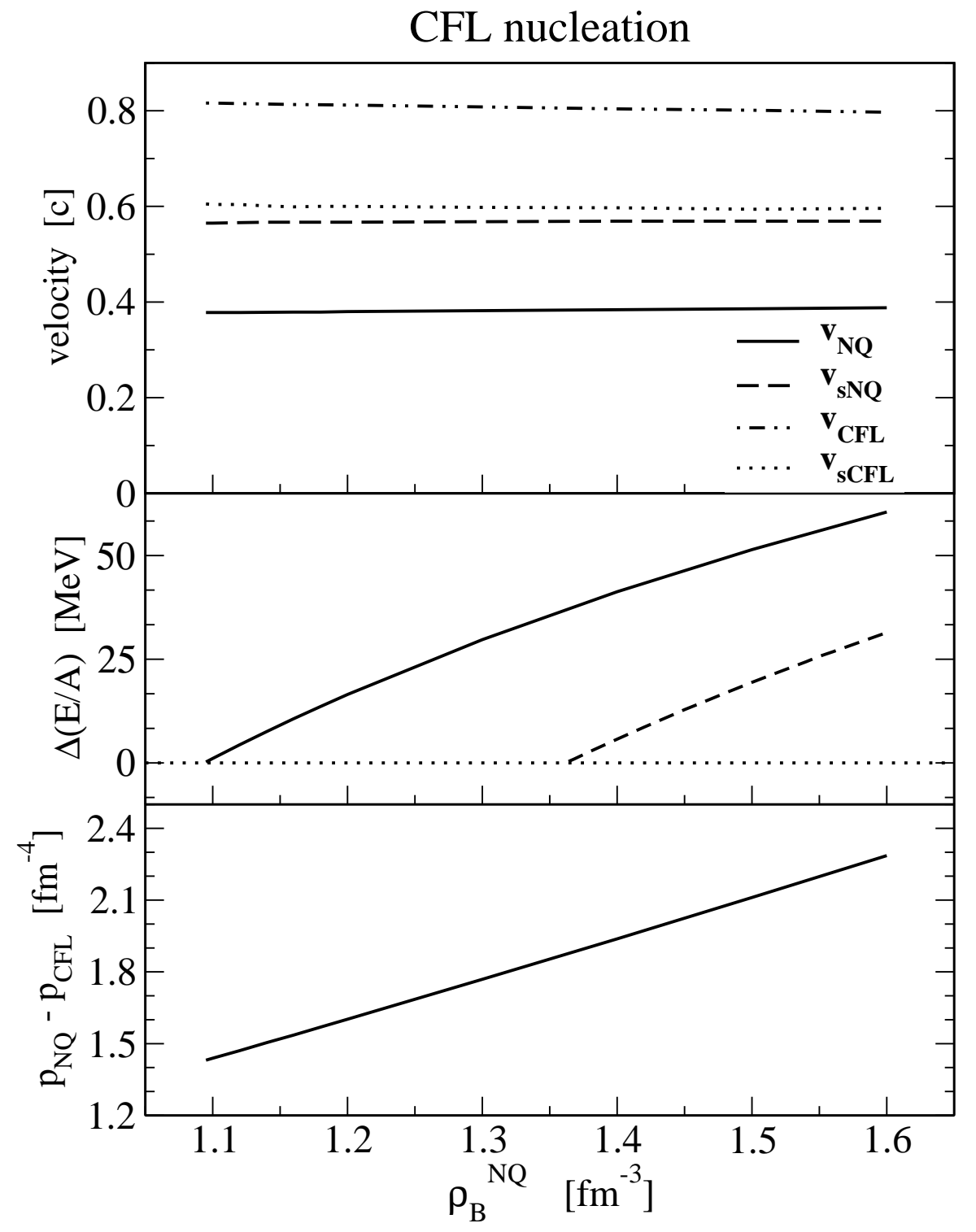

Fig. 19. - Results for the conversion front separating $\beta$-stable quark matter $\left(B^{1 / 4}=155\right.$ $\mathrm{MeV}$ and $\left.B^{1 / 4}=165\right)$ from gapped quark matter $(\Delta=100 \mathrm{MeV})$. Upper panel: velocity of the Normal Quark phase $v_{\mathrm{NQ}}$, of the diquark condensed CFL phase $v_{\mathrm{CFL}}$ and corresponding sound velocities $v_{\mathrm{sNQ}}$ and $v_{\mathrm{sCFL}}$, all in units of the velocity of light and in the front frame. Here for simplicity only the results for $B^{1 / 4}=155$ are presented. Center panel: energy difference between the two phases (in the NQ phase rest frame). Here we show the results both for $B^{1 / 4}=155 \mathrm{MeV}$ (solid line) and for $B^{1 / 4}=165 \mathrm{MeV}$ (dashed line). Lower panel: pressure difference between the two phases. The difference between the two pressures is almost exactly $B$ independent. 


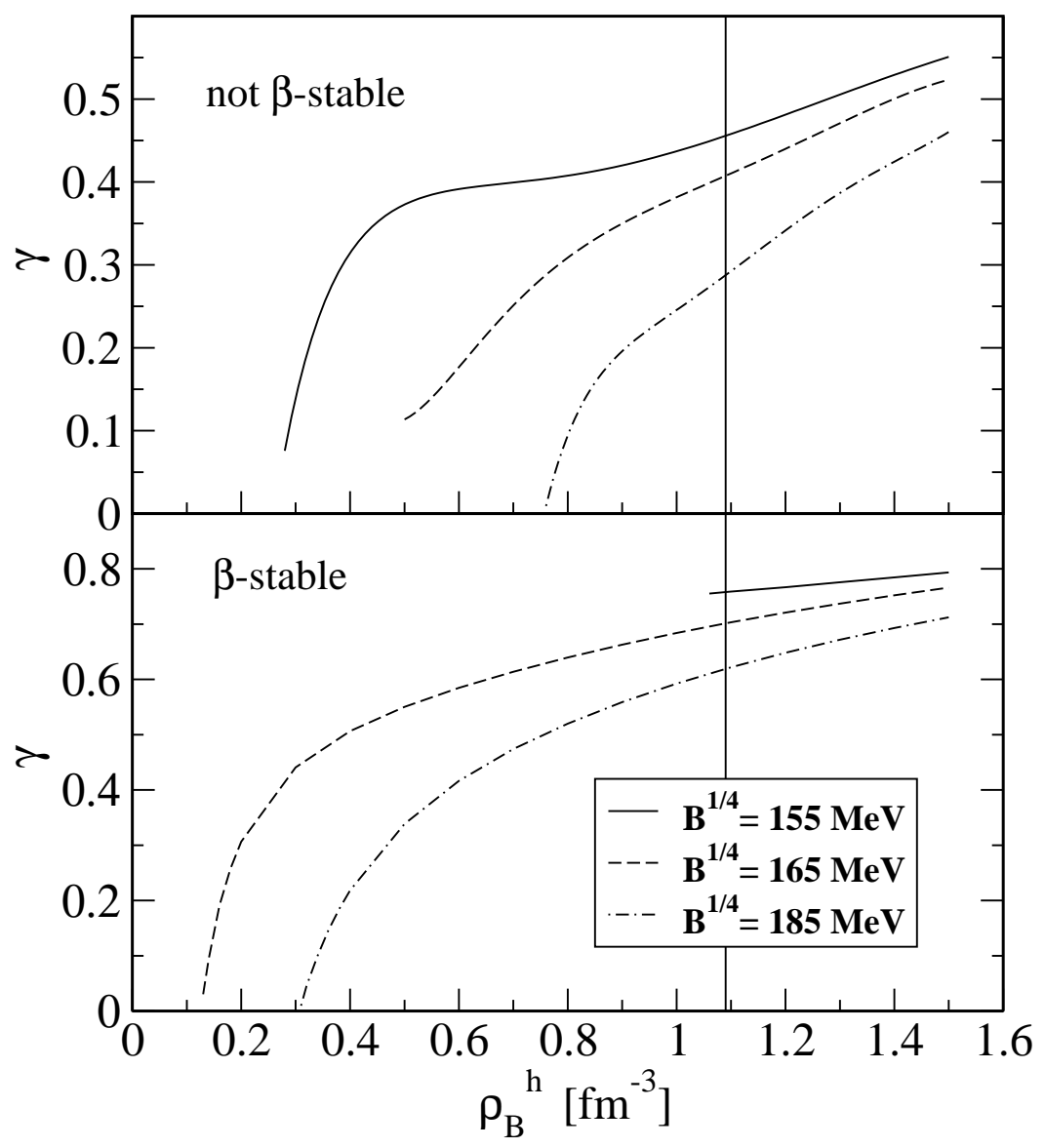

Fig. 20.- The $\gamma$-factor entering the fractal dimension of the conversion front, as a function of the baryonic density of the hadronic phase. The vertical line corresponds to the central density of the most massive stable configuration of a nucleonic star obtained using GM3 model $\left(\rho_{h}^{\max }=1.09 \mathrm{fm}^{-3}\right)$. 


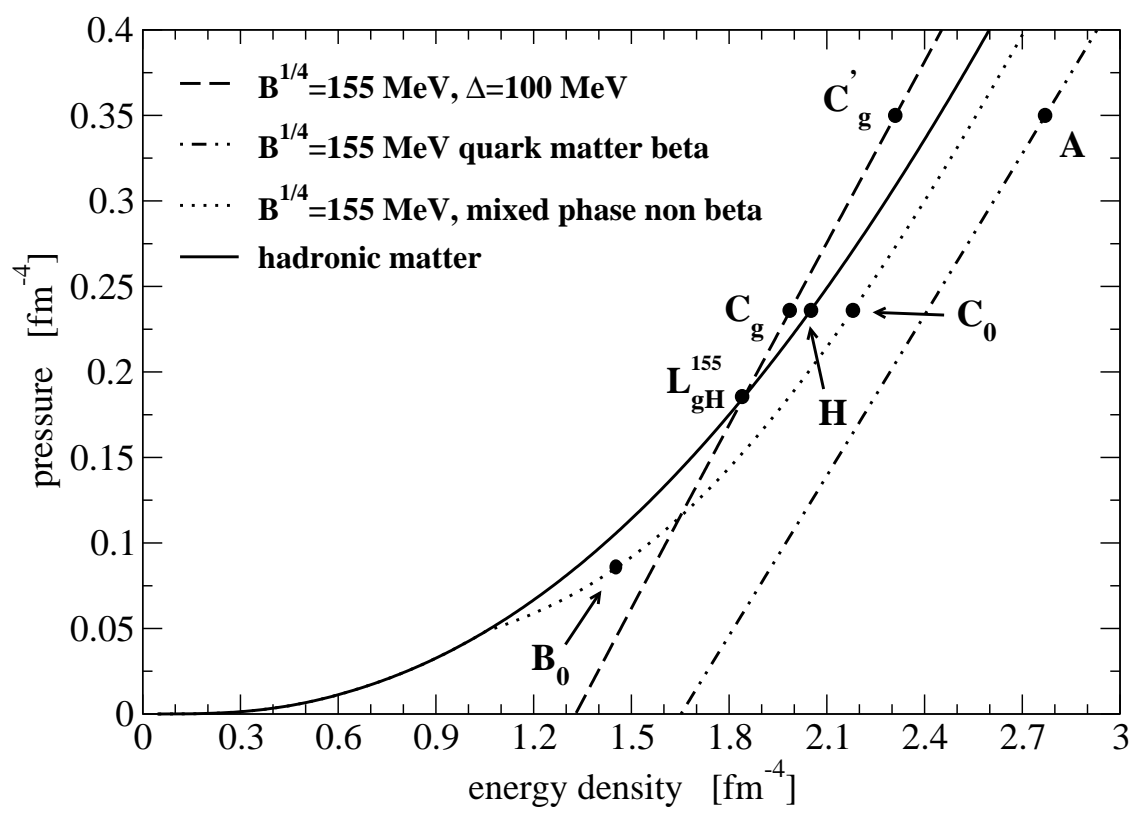

Fig. 21. - Scheme for convection: $\mathrm{H}$ represents the drop of hadronic matter just before deconfinement, $\mathrm{B}_{0}$ represents the drop of newly formed QM, C stays for the drop of QM after pressure equilibration and $\mathrm{L}$ indicates the end point of the convective layer. Finally A represents a drop of ungapped quark matter before its transition to CFL phase. Here $B^{1 / 4}=155 \mathrm{MeV}$ and hyperons are not included.

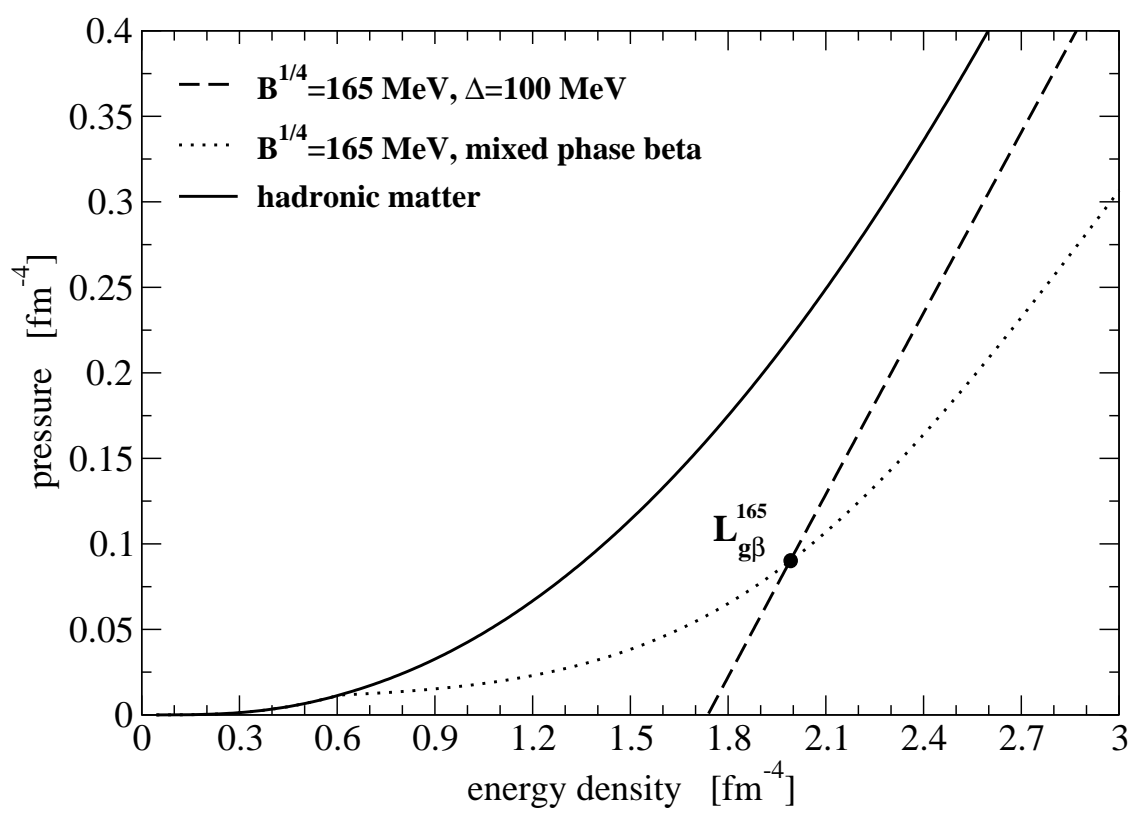

Fig. 22. - Scheme for convection: see Fig. 21. Here $B^{1 / 4}=165 \mathrm{MeV}$ and hyperons are not included. 


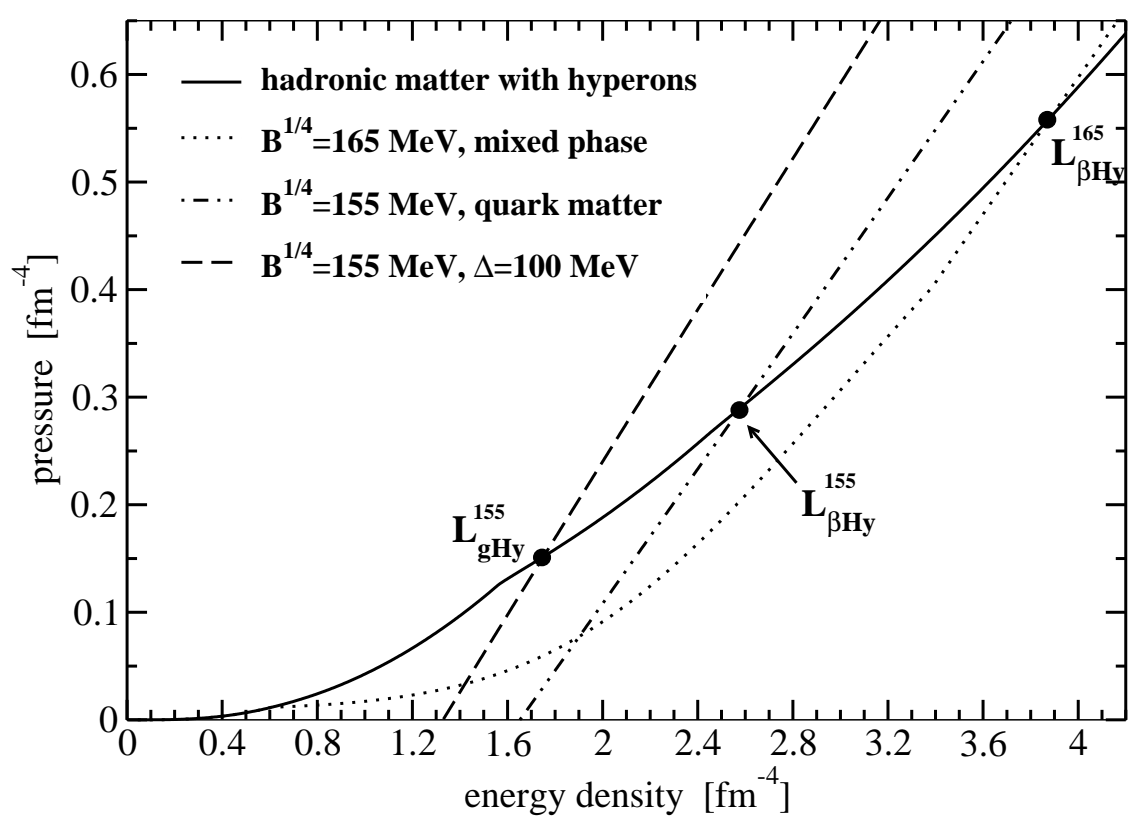

Fig. 23. - Scheme for convection: see Fig. 21. Here $B^{1 / 4}=155 \mathrm{MeV}$ and $165 \mathrm{MeV}$ and hyperons are included. 